\title{
Oral treatment with the herbal formula B307 alleviates cardiac toxicity in doxorubicin-treated mice via suppressing oxidative stress, inflammation, and apoptosis
}

\author{
This article was published in the following Dove Press journal: \\ OncoTargets and Therapy \\ 26 May 2015 \\ Number of times this article has been viewed
}

\section{Chia-Ying Lien,' Tai-Yuan Chuang,' Chih-Hsiang Hsu, ${ }^{2}$ Ching-Lung Lin, ${ }^{2}$ Sheue-Er Wang, ${ }^{2}$ Shuenn-Jyi Sheu, ${ }^{3}$ Chiang-Ting Chien, ${ }^{2}$ Chung- $\mathrm{H} \sin \mathrm{Wu}^{2}$ \\ 'Department of Athletics, National Taiwan University, Taipei, Taiwan; \\ ${ }^{2}$ Department of Life Science, National Taiwan Normal University, Taipei, Taiwan; ${ }^{3}$ Brion Research Institute of Taiwan, New Taipei City, Taiwan}

Video abstract

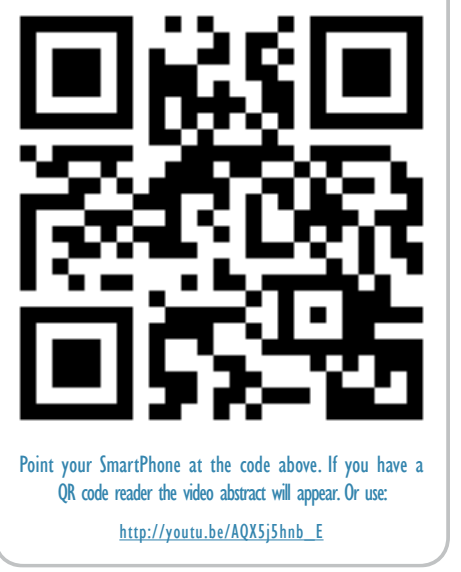

Correspondence: Chung-Hsin Wu Department of Life Science, National Taiwan Normal University, No 88, Tingzhou Road, Section 4, Wenshan District, Taipei I 1677, Taiwan

Tel +886277346363

Fax +8862 2931 2904

Email megawu@ntnu.edu.tw
Objective: This study aimed to investigate whether the herbal formula B307 could alleviate doxorubicin (DOX)-induced acute cardiotoxicity. If so, we further unraveled possible molecular mechanisms of cardiac protection under treatment with the herbal formula B307.

Methods: Before the animal experiment, we examined relative viabilities of Huh7 cancer cells under treatment with the herbal formula B307. To test whether oral treatment with the herbal formula B307 could alleviate cardiotoxicity, equal volumes of B307 (50 mg/kg) or saline (sham treatment) were administered to 20 -week-old male mice once daily for 14 consecutive days. Then, DOX (10 mg/kg; ip) was administered to male mice under B307 and sham treatments at 22-23 weeks of age. Cardiac functions in these mice were assessed via echocardiography at 23-24 weeks of age. Then, expressions of oxidative stress, inflammation, and apoptosis-related proteins were examined in the heart tissue by immunohistochemistry and Western blotting at 24-25 weeks of age. Apart from this, mortality rate and body weight were measured during the experiment.

Results: In vitro, the relative viabilities of Huh7 cancer cells under treatment with the herbal formula B307 had shown no obvious change at doses of 10-160 ng/mL. Furthermore, the relative viabilities of Huh7 cancer cells were significantly reduced under DOX treatment but showed no significant change under DOX only and DOX plus B307 treatment. In vivo, the mortality rate, body weight, and cardiac function of DOX-treated mice were obviously improved under oral treatment with the herbal formula B307. Furthermore, cardiac expressions of endothelial nitric oxide synthase, superoxide dismutase 2, and B-cell lymphoma 2 were significantly enhanced, but tumor necrosis factor alpha, NFKB1 (p50 and its precursor, p105), neurotrophin-3, Bcl-2associated $X$ protein, calpain, caspase 12 , caspase 9 , and caspase 3 were significantly suppressed in DOX-treated mice under oral treatment with the herbal formula B307.

Conclusion: Our results revealed that oral treatment with the herbal formula B307 may provide cardioprotection in DOX-treated mice via suppressing oxidative stress, inflammation, and apoptosis in heart tissue. We believe that the herbal formula B307 may be developed as a potential alternative treatment for cancer patients under DOX treatment.

Keywords: doxorubicin, cardiac protection, oxidative stress, inflammation, apoptosis, Chinese herbal formula, mouse model

\section{Introduction}

Doxorubicin (DOX) is an effective chemotherapeutic agent in treating human neoplasms such as leukemia, lymphomas, and solid tumors. ${ }^{1}$ However, DOX often causes irreversible degenerative cardiomyopathy and congestive heart failure. ${ }^{2}$ Acute cardiotoxicity 
has been recognized as a severe complication with DOX chemotherapy. ${ }^{3}$ Previous studies have shown that oxidative stress, inflammation, and apoptosis might be involved in the pathogenesis of DOX-induced cardiotoxicity. ${ }^{4}$ Furthermore, it has been suggested that DOX may induce the generation of reactive oxygen species (ROS), which then leads to oxidative damage of cellular and mitochondrial membranes. ${ }^{5,6}$ To find a potential reliever that can protect from cardiotoxicity, yet not interfere with the chemotherapeutic effect, will be very urgent for cancer patients under DOX treatment.

Nowadays, many studies have suggested that some natural extracts may alleviate acute cardiotoxicity in DOX-treated animals. For example, there is a recent study showing that chrysin (5,7-dihydroxyflavone) has a potent protective effect against DOX-induced acute cardiotoxicity in rats via suppressing oxidative stress, inflammation, and apoptotic tissue damage. ${ }^{7}$ Chrysin has been found in bee propolis, honey, and various plants. ${ }^{8}$ In Taiwan, the herbal formula B307 has been widely used to enhance cardiovascular function and muscle strength. The main herbal ingredients of the herbal formula B307 are Ginseng (Panax ginseng Radix) and Danshen (Salviae Miltiorrhizae Radix). Ginseng is widely used as a traditional herbal medicine that acts multifunctionally as an antioxidant, anti-inflammatory, and altering agent in the expression of neurotrophic factors. ${ }^{9-14}$ In addition, Danshen is widely used to treat heart disease and ameliorate an atherosclerosis effect in humans and rodents. ${ }^{15-17}$ The herbal formula B307 may be a potential candidate reliever for cancer patients under DOX treatment. Thus, we aimed to investigate the cardioprotective effects of oral B307 treatment in DOX-treated mice.

In this study, we compared mortality rate, body weight, cardiac function, and microcirculation between DOX-treated mice under oral B307 and sham treatments. By immunohistochemistry and Western blotting, we examined and compared cardiac expressions of oxidative stress, inflammation, and apoptosis-related proteins between DOX-treated mice under oral B307 and sham treatments. Our study revealed that the herbal formula B307 might be developed as a potential reliever for cancer patients undergoing DOX chemotherapy.

\section{Materials and methods}

\section{Chromatographic fingerprint analysis of the herbal formula B307}

The herbal formula B307 (supplied by Sun-Ten Pharmaceutical Company, New Taipei City, Taiwan) mainly contains ingredients of Ginseng Radix, Schizandrae Fructus,
Ophiopogonis Tuber, and Salviae Miltiorrhizae Radix. All chemical compounds used in this analysis were dissolved in distilled water $\left(\mathrm{H}_{2} \mathrm{O}\right)$ /methanol $(\mathrm{MeOH})$. The chromatographic fingerprint analysis was conducted using liquid chromatography-mass spectrometry (LC/MS) analysis. Fifteen bioactive marker substances were qualitatively determined within 80 minutes under a selected LC/MS condition, as shown in Figure 1. The LC/MS analytical system consisted of a Shimadzu LC-20AD UFLC system linked with a LCMS-8040 triple quadrupole mass spectrometer. The UFLC condition was set as follows: gradient elution by the mixture of mobile phases A $(0.1 \%$ formic acid and $1 \mathrm{~g} / \mathrm{L}$ solution of ammonium acetate in $\left.\mathrm{H}_{2} \mathrm{O}\right)$ and $\mathrm{B}(0.1 \%$ formic acid and $1 \mathrm{~g} / \mathrm{L}$ solution of ammonium acetate in $\mathrm{MeOH}$ ) at minutes $0-40$ with the ratio of $100 \%-70 \% \mathrm{~A}$ and $0 \%-30 \% \mathrm{~B}$; at minutes $40-70$ with the ratio of $70 \%-0 \% \mathrm{~A}$ and $30 \%-100 \% \mathrm{~B}$; at minutes $70-70.1$ with the ratio of $0 \%-100 \% \mathrm{~A}$ and $100 \%-0 \% \mathrm{~B}$; and at minutes $70.1-80$ with the ratio of $100 \% \mathrm{~A}$ and $0 \% \mathrm{~B}$. The flow rate was $0.4 \mathrm{~mL} / \mathrm{min}$; the column temperature was kept at $40^{\circ} \mathrm{C}$; the injection volume was $20 \mu \mathrm{L}$; and the analytical column was a Shimadzu Shim-pack XR-ODS II column $(2.2 \mu \mathrm{m}, 2 \times 100 \mathrm{~mm}$, Shimadzu). Dual ion modes [electrospray ionization, $\operatorname{ESI}(+)$ and $\operatorname{ESI}(-)$ ] were used in MS detection, and the transmission of $[\mathrm{M}+\mathrm{H}]^{+}$and $[\mathrm{M}-\mathrm{H}]^{-}$ was set as the optimum condition. The MS detection was set as a full scan range (100-1,200 amu); the interface voltages were set at $4.5 \mathrm{kV}$ for $\operatorname{ESI}(+)$ and $-3.5 \mathrm{kV}$ for $\operatorname{ESI}(-)$. Nitrogen as a nebulizing and drying gas, the flow was at 3.0 and $10 \mathrm{~L} / \mathrm{min}$, respectively. Argon as a CID gas was set at $230 \mathrm{kPa}$. DL temperature was at $150^{\circ} \mathrm{C}$, whereas heat block temperature was at $400^{\circ} \mathrm{C}$.

\section{Cell viability of Huh7 cancer cells treated with the herbal formula B307 and DOX}

Cell viability of Huh7 cancer cells was measured as described in our previous study. ${ }^{18}$ All experiments in vitro were conducted in a laboratory environment. We cultured Huh7 cancer cells (human hepatoma cells) in culture flasks $\left(25 \mathrm{~cm}^{2}\right)$. Then, the Huh7 cells were maintained in Dulbecco's Modified Eagle's Medium (DMEM, WelGene, Daegu, Korea), seeded on cell culture dishes (Falcon, San Jose, CA, USA), and subcultured by trypsin-EDTA (GibcoBRL, Grand Island, NY, USA) treatment. All Huh7 cells were maintained at $37^{\circ} \mathrm{C}$ in a humidified atmosphere $\left(95 \%\right.$ air and $\left.5 \% \mathrm{CO}_{2}\right)$. Afterward, the Huh7 cells were cultured with and without the herbal formula B307 (50-100 ng/mL dissolved in dimethyl sulfoxide) for 1 hour and then treated with DOX $(10-40 \mu \mathrm{g} / \mathrm{mL})$ for 24 hours. Control cultures were also administered the same 

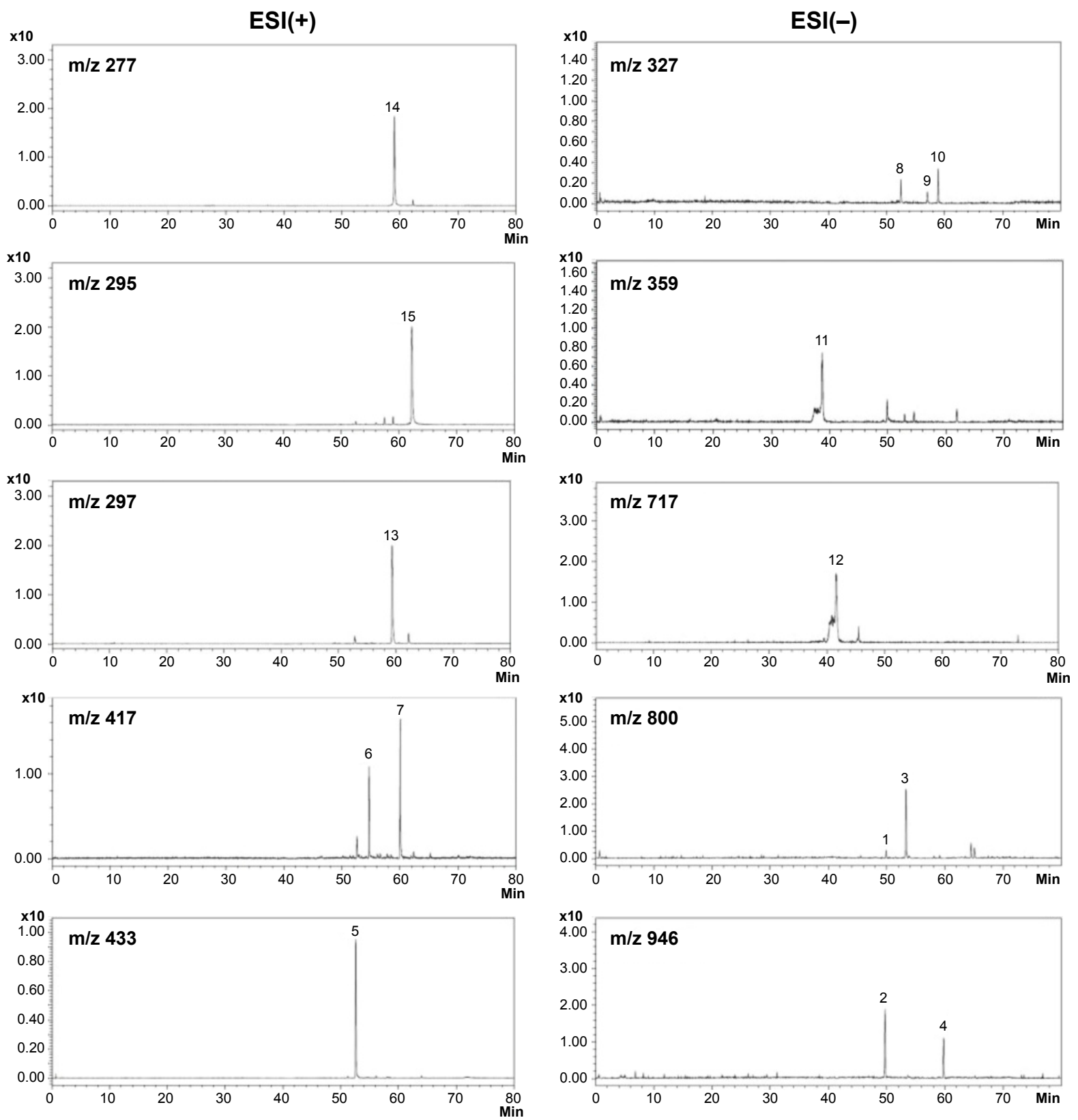

Figure I Chromatographic fingerprint analysis for the herbal formula B307.

Notes: Chromatographic fingerprint analysis was conducted using LC/MS analysis. Fifteen bioactive marker substances from ingredients of the herbal formula B307 were qualitatively determined within 80 minutes under the selected LC/MS condition. Bioactive marker substances for Ginseng Radix: Ginsenoside RgI (peak I), Ginsenoside Re (peak 2), Ginsenoside Rf (peak 3), Ginsenoside Rd (peak 4), Schizandrae Fructus: Schizandrin (peak 5), Gomisin A (peak 6), Deoxyschizandrin (peak 7), Ophiopogonis Tuber: Ophiopogonanone A (peak 8), Methylophiopogonone A (peak 9), Methylophiopogonanone B (peak I0), Salviae Miltiorrhizae Radix: rosmarinic acid (peak II), salvianolic acid B (peak I2), cryptotanshinone (peak I3), tanshinone I (peak I4), and tanshinone IIA (peak I5).

Abbreviations: AU, arbitrary perfusion units; LC/MS, liquid chromatography-mass spectrometry; ESI, electrospray ionization; m/z, mass-to-charge ratio.

amount of DMEM. After the treatment with B307 and DOX, we added $0.5 \mathrm{mg} / \mathrm{mL}$ MTT to the culture media. Then, the Huh7 cells with DOX treatment were incubated for 3 hours at $37^{\circ} \mathrm{C}$. Absorbance was read at an optical density (OD) of $570 \mathrm{~nm}$ with an enzyme-linked immunosorbent assay reader (uQuant, BioTek, Inc., Winooski, VT, USA). The percentage of Huh7 cell viability was calculated as OD at $570 \mathrm{~nm}$ of the experiment well divided by OD at $570 \mathrm{~nm}$ of the control well.
Cell viability of the Huh7 cancer cells with vehicle, DOX, and B307 treatments was repeated at least six times under each condition.

\section{Oral B307 treatments and ethical approval of animal research}

In this study, we chose 20-week-old ICR mice to feed either B307 extract $(30 \mathrm{mg} / \mathrm{mL}$, the $\mathrm{pH}$ value was close to 7.0 ) 
or their vehicle (dimethyl sulfoxide) in their drinking water twice daily for 14 days. The mice were then treated with two intraperitoneal injections of DOX (10 mg/kg body weight). The DOX injection was separated by 3 days. The mice were housed with three to six per cage (unless otherwise specified) and maintained on a 12:12 hour light-dark cycle with water and food ad libitum. Body weights of mice were monitored daily. All the mice in this study were checked daily for determining lifespan. Their death judgment rests on cardiac arrest. Mice euthanasia would be carried out under the circumstance of moribund, including lack of movement even after prodding, and/or lying on side, and lack of righting reflex. All protocols in the experiment was executed according to the international guidelines for care and use of laboratory animals. In the experiment, all animals were maintained according to the procedures outlined in the National Institute of Health Guide for the Care and Use of Laboratory Animals. Our use of laboratory animals has been approved by the Committee on Animal Research of National Taiwan Normal University (Protocol number: NTNU/Animal Use/No 103012/June 25, 2014).

\section{Cardiovascular function}

Cardiovascular function of mice at the age of 23-24 weeks was assessed under sham, DOX, and B307 plus DOX (B307+DOX) treatments individually by echocardiography. The M-mode echocardiography was performed with the mice under isoflurane inhalation anesthesia to measure cardiac parameters using a prospect high-resolution imaging system (S-Sharp Corporation, New Taipei City, Taiwan), with the probe providing the central frequency at $40 \mathrm{MHz}$. After anesthesia, the mice were placed in a sealed box and exposed to a $2 \%$ isoflurane gas (Baxter Healthcare, New Providence, RI, USA) at a flow rate of $2 \mathrm{~L} / \mathrm{min}$. Then, each one was placed on a heated working platform for monitoring ECG and respiration gating. Both the anterior and left lateral thoracic regions of the mice were shaved. US gel (Home Care Technology Co., Ltd, Tainan, Taiwan) was then coated as a coupling agent on the skin. The PB406 transducer was pasted to get left ventricular M-mode images. The wall thickness and fractional shortening was determined by M-mode images. Mitral and aortic blood flow velocities were measured from an apical view. Changes of heart rates (HRs), left ventricular fractional shortening (LVFS), ejection fraction (EF), stroke volume (SV), cardiac output (CO), maximal mitral valve blood flow velocity (MV max), and maximal aortic valve blood flow velocity (AV max) were measured and compared among these mice. For echocardiographic measures, at least three stable consecutive cardiac cycles were selected and averaged for each mouse.

\section{Subcutaneous microcirculation measurement}

As shown in the previous study, we used a Laser-Doppler imager (Moor Instruments, Axminister, UK) to scan regional dermal microvascular blood flow in the mice at 23-24 weeks of age under sham, B307, DOX, and B307+DOX treatments individually. ${ }^{19}$ The camera of the Laser-Doppler imager was positioned at $18 \mathrm{~cm}$ above the skin of the mice. Then, the blood flow of the mice was recorded and analyzed by using data acquisition measurement software (moorFLPI version V3.0, Moor Instruments) in arbitrary perfusion units. For skin blood flow measurements, at least three stable consecutive Laser-Doppler images were selected and averaged for each mouse.

\section{ROS analysis}

As in the previous study, luminol chemiluminescence (CL) was used to measure free radical formation. ${ }^{20}$ We measured blood ROS in the mice at 23-24 weeks of age under sham, B307, DOX, and B307+DOX treatments individually. The blood of the mice was phlebotomized, and then blood samples $(200 \mu \mathrm{L})$ were put on the measurement plate with stir as background for 1 minute. The samples were then mixed with $0.5 \mathrm{~mL}$ of $0.2 \mathrm{mM}$ luminol sodium salt (Sigma A4685, Sigma-Aldrich Corporation, St. Louis, MO, USA) to continually detect signals with an integration time of 10 seconds for 3 minutes. For ROS analysis, at least three stable consecutive ROS values were selected and averaged for each mouse.

\section{Cardiac immunohistochemistry}

The mice at 24-25 weeks of age under sham, B307, DOX, and B307+DOX treatments were anesthetized and cardiac perfused with phosphate-buffered saline containing $4 \%$ formaldehyde individually. We removed cardiac tissue from the mice and fixed it with 4\% formaldehyde. Cardiac specimens were embedded in paraffin and cut into tissue sections at a thickness of $5 \mu \mathrm{m}$. The tissue sections were mounted on slides for histological and immunohistochemical (IHC) analysis. General cardiac morphology and extent of cardiac fibrosis deposition were assessed by using H\&E staining. By the heatinduced epitope retrieval method, cardiac tissue sections were separately stained at room temperature for 1 hour with antibodies of endothelial nitric oxide synthase (eNOS), nuclear factor- $\kappa \mathrm{B}(\mathrm{NF}-\kappa \mathrm{B})$, tumor necrosis factor alpha (TNF- $\alpha)$, superoxide dismutase 2 (SOD2), neurotrophin-3 (3-NT), 
calpain, caspase 12, caspase 9, caspase 3 (Cell Signaling Technology, Inc., Danvers, MA, USA), B-cell lymphoma 2 (Bcl-2, Santa Cruz Biotechnology, Inc., Santa Cruz, CA, USA), Bcl-2-associated X protein (Bax, Thermo Fisher Scientific, Inc., Waltham, MA, USA), and cytochrome C (Cyto-C, Abcam, Inc., Cambridge, MA, USA). As immunostaining controls for each antibody, the serial $5 \mu \mathrm{m}$ crosssections were treated with the unanimous staining protocol. Immunostaining detection was executed by incubation with biotinylated secondary antibodies (Novolink ${ }^{\mathrm{TM}}$ polymer detection system 1) at room temperature for 30 minutes, then by incubation with an avidin-biotin-HRP complex (Novolink ${ }^{\text {TM }}$ Polymer Detection System 1) for 30 additional minutes. Immunostaining visualization was performed with DAB Chromogen (Novolink ${ }^{\mathrm{TM}}$ polymer detection system 1) and counterstained with hematoxylin (Novolink ${ }^{\mathrm{TM}}$ polymer detection system 1) following the supplier's protocol.

\section{Cardiac Western blotting}

The removed heart tissue was homogenized in a buffer solution that included $0.05 \mathrm{M}$ tris(hydroxymethyl)aminomethane (pH 8.0, Bionovas, Inc., Washington, DC, USA), $0.15 \mathrm{M}$ sodium chloride, $0.02 \mathrm{M}$ ethylenediaminetetraacetic acid, 1\% deoxycholic acid, 1\% nonidet P40 (Bionovas, Inc.), $0.1 \%$ sodium dodecyl sulfate (SDS, Bionovas, Inc.), $1 \%$ protease inhibitor cocktail for full range, $1 \%$ serine/threonine phosphatase inhibitor cocktail, and 1\% tyrosine phosphatase inhibitor cocktail (Bionovas, Inc.). The homogenized buffer solution was placed on ice for 1 hour and then centrifuged at $4^{\circ} \mathrm{C}$ for $13,000 \mathrm{rpm}$ for another 20 minutes. The supernatant solution was then separated. The separated solution was quantitated by using a BCA protein assay kit (Thermo Fisher Scientific, Inc.). In all, $30 \mathrm{mg}$ of the total protein was denatured at $95^{\circ} \mathrm{C}$ for 5 minutes with $5 \mathrm{X}$ sample dye, which included 0.25 M tris(hydroxymethyl) aminomethane hydrochloride ( $\mathrm{pH} 6.8$, Bionovas, Inc.), $10 \%$ SDS, $0.5 \%$ bromophenol blue (Bionovas, Inc.), $50 \%$ glycerol, and 5\% $\beta$-mercaptoethanol (Bionovas, Inc.). The electrophoresis was done using a $12.5 \%$ discontinuous sodium dodecylsulfate-polyacrylamide gel. The proteins were then electroblotted onto a $0.2-\mu \mathrm{m}$ polyvinylidene difluoride (PVDF, GE Healthcare Life Sciences, Barrington, IL, USA) membrane for 120 minutes at $100 \mathrm{~V}$. The membranes were allowed to react with a blocking buffer $(5 \%$ skim milk in TBS-T buffer) for 1 hour at the ambient temperature, and then they were blocked.

The antibodies of eNOS, NF-кB, TNF- $\alpha$, SOD2, 3-NT, calpain, caspase 12 , caspase 9 , caspase 3 (Cell Signaling
Technology, Inc.), Bcl-2 (Santa Cruz Biotechnology, Inc.), $\beta$-actin, Bax (Thermo Fisher Scientific, Inc.), and Cyto-C (Abcam, Inc., Cambridge, MA, USA) were allowed to react for 2 hours at the ambient temperature (or overnight at $4^{\circ} \mathrm{C}$ ). The membrane was washed three times using TBS-T at intervals of 10 minutes. Then the secondary antibodies, anti-rabbit IgGHRP (1:5,000 dilution, PerkinElmer, Inc., Waltham, MA, USA), anti-mouse IgG-HRP (1:5,000 dilution, PerkinElmer, Inc.), and anti-goat IgG-HRP (1:5,000 dilution, Enzo Life Sciences, Inc., Farmingdale, USA), were allowed to react at the ambient temperature for 1 hour. The membrane was washed again with TBS-T three times at an interval of 10 minutes between each washing. Each band was made visible by utilizing ECL Western blotting detection reagents (GE Healthcare Life Sciences), and the chemiluminescence was detected using LAS-4000 (GE Healthcare Life Sciences). Densitometric assessments of the bands were performed using ImageJ software (version 1.48t, Wayne Rasnabd, Washington, DC, USA).

\section{Statistical analysis}

All data were given as mean \pm standard error of the mean from at least six independent experiments. The number of mice under sham, B307, DOX, and B307+DOX treatments was at least six for each group. Statistical analysis was performed using a two-way analysis of variance followed by a Student-Newman-Keuls multiple comparisons posttest. The $P$-values of at least $<0.05$ were considered significant.

\section{Results}

\section{Chromatographic fingerprint of the herbal formula B307}

Chromatographic fingerprint analysis using LC/MS analysis for ingredients of the herbal formula B307 is shown in Figure 1. Fifteen bioactive marker substances were qualitatively determined within 80 minutes under the selected LC/MS condition. We observed that the herbal formula B307 mainly contains ingredients of Ginseng Radix, Schizandrae Fructus, Ophiopogonis Tuber, and Salviae Miltiorrhizae Radix. Chromatographic fingerprint analysis was conducted using LC/MS analysis. Fifteen bioactive marker substances from ingredients of the herbal formula B307 were qualitatively determined within 80 minutes under the selected LC/MS condition. Bioactive marker substances for Ginseng Radix were Ginsenoside Rg1, Ginsenoside Re, Ginsenoside Rf, and Ginsenoside Rd; marker substances for Schizandrae Fructus were Schizandrin, Gomisin A, and Deoxyschizandrin; marker substances for Ophiopogonis Tuber were Ophiopogonanone A, 
Methylophiopogonone A, and Methylophiopogonanone B; marker substances for Salviae Miltiorrhizae Radix were Rosmarinic acid, Salvianolic acid B, Cryptotanshinone, Tanshinone I, and Tanshinone IIA.

\section{Cell viability of Huh7 cancer cells under sham, DOX, and B307+DOX treatment}

Figure 2A shows cell viabilities of Huh7 cancer cells under B307 treatment with different doses. Our data had shown that cell viability of Huh7 cancer cells was not significant under B307 treatment at $10-160 \mathrm{mg} / \mathrm{mL}(P>0.01)$. Figure $2 \mathrm{~B}$ shows the effect of DOX in Huh7 cancer cells under sham, DOX, and B307+DOX treatments. We repeated cell viability testing of Huh7 cancer cells with treatments of vehicle, DOX, and B307 at least six times under each condition. Our data had shown that cell viability of Huh7 cancer cells was significantly reduced by approximately $50 \%-60 \%$ under DOX treatment at $10-30 \mathrm{mg} / \mathrm{mL}(P<0.01)$. Furthermore, the cell viability of DOX-treated Huh7 cancer cells was significantly reduced 50\%-60\% under B307 treatment at $50-100 \mathrm{ng} / \mathrm{mL}(P<0.01)$. There was no observable difference between cell viability of Huh7 cancer cells under DOX only and DOX+B307 treatment $(P>0.05)$.

\section{Survival rates and body weight of mice under sham, DOX, and B307+DOX treatment}

Figure 3A shows survival rates of the mice under sham, DOX, and B307+DOX treatments. The mice were orally treated with either B307 extract or their vehicle twice daily at 20-22 weeks of age, and they were then treated with two intraperitoneal injections of DOX within a week. Our results had shown that survival rates of mice began to reduce following DOX treatment and thereafter. The survival rate of the mice under DOX treatment at 30 weeks of age was reduced to $50 \%$. However, the mice under sham and B307+DOX treatments were still alive.

Figure $3 \mathrm{~B}$ shows the body weight of the mice under treatments of sham, DOX, and B307+DOX. We observed that the body weight of the mice began to reduce following DOX treatment and thereafter. The body weight of DOX-treated mice was significantly lower than those of the mice under sham treatment from 24 weeks of age and thereafter (DOX group vs sham group, $P<0.01$ ), whereas the body weight of DOX-treated mice under oral B307 treatment was significantly higher than DOXtreated mice from 24 weeks of age and thereafter (B307+DOX group vs DOX group, $P<0.01-0.05)$. Furthermore, we also observed that the body weight of DOX-treated mice under oral B307 treatment was significantly lower than those of the mice under sham treatment from 26 weeks of age and thereafter (B307+DOX group vs sham group, $P<0.01-0.05$ ).

\section{Cardiac function of mice under sham, DOX, and B307+DOX treatment}

Figure 4A shows an example of systolic and diastolic cardiac functions in the mice at 23-24 weeks of age under sham, DOX, and B307+DOX treatments by non-invasive echocardiography. We measured and then quantified cardiac function in HRs, LVFS, EF, SV, CO, MV max, and AV max in the mice from echocardiographic evidence in Figure 4A. We observed that HRs, LVFS, EF, SV, CO, MV max, and AV max in the DOX-treated mice were significantly lower than those of the mice under sham treatment (DOX group vs sham group, HR: $252 \pm 25$ vs $327 \pm 27$ bpm, $P<0.01$; FS: $31.2 \% \pm 5.1 \%$ vs $50.5 \% \pm 5.4 \%, P<0.01$; EF: $56.1 \% \pm 8.5 \%$ vs $98.5 \% \pm 11.2 \%$, $P<0.01 ; \mathrm{SV}: 37.2 \pm 3.5$ vs $55.6 \pm 4.4 \mu \mathrm{L}, P<0.01 ; \mathrm{CO}$ : $15.3 \pm 1.4$ vs $20.2 \pm 2.1 \mathrm{~mL} / \mathrm{min}, P<0.01$; MV max: $35.5 \pm 8.3 \mathrm{vs}$
A

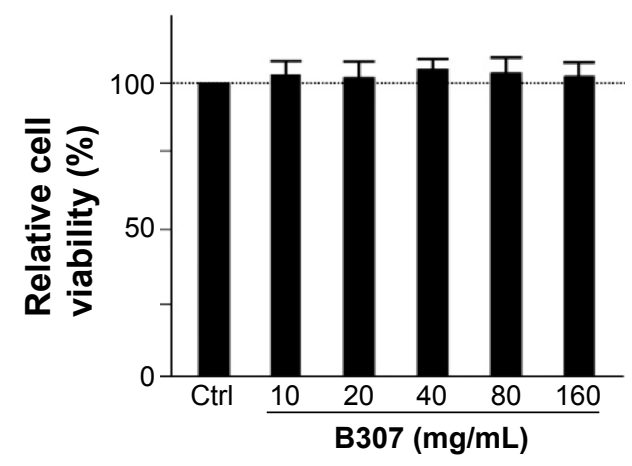

B

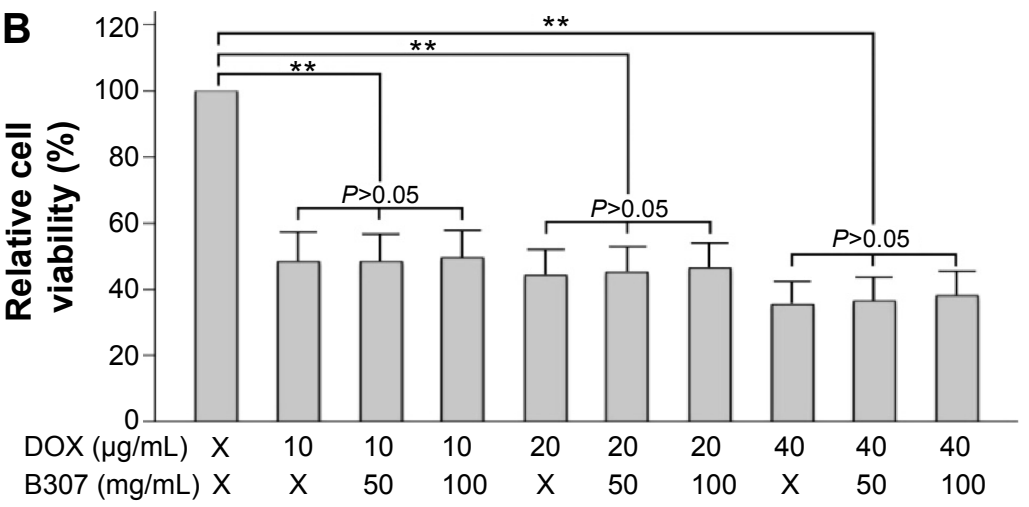

Figure 2 Viability of Huh7 cancer cell under sham, DOX, and B307+DOX treatments.

Notes: (A) The relative viabilities of Huh7 cancer cells were not changed under B307 treatment at doses of $10-160 \mathrm{mg} / \mathrm{mL}$. (B) The relative viabilities of Huh7 cancer cells were significantly reduced under DOX treatment but were not significant between DOX only and DOX+B307 treatment. Each treatment was repeated at least six times. Values are mean \pm SEM ( $* * P<0.01$, two-way ANOVA followed by a Student-Newman-Keuls multiple comparisons posttest).

Abbreviations: DOX, doxorubicin; MW, molecular weight; SEM, standard error of the mean; ANOVA, analysis of variance. 
A

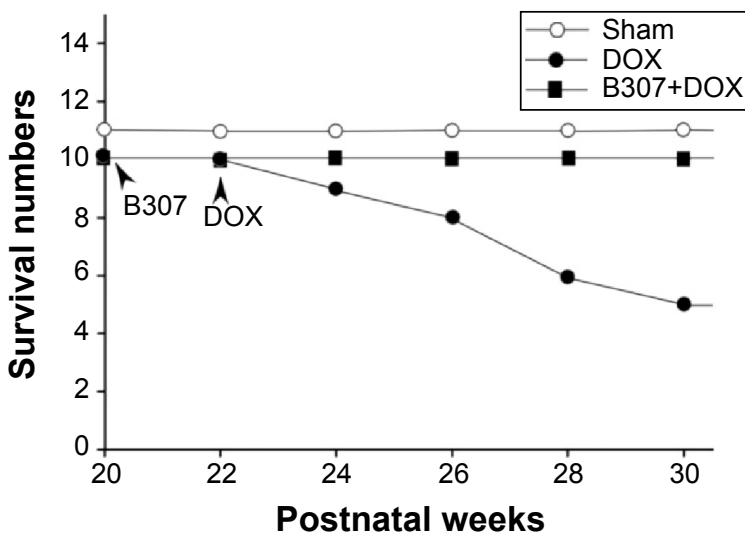

B

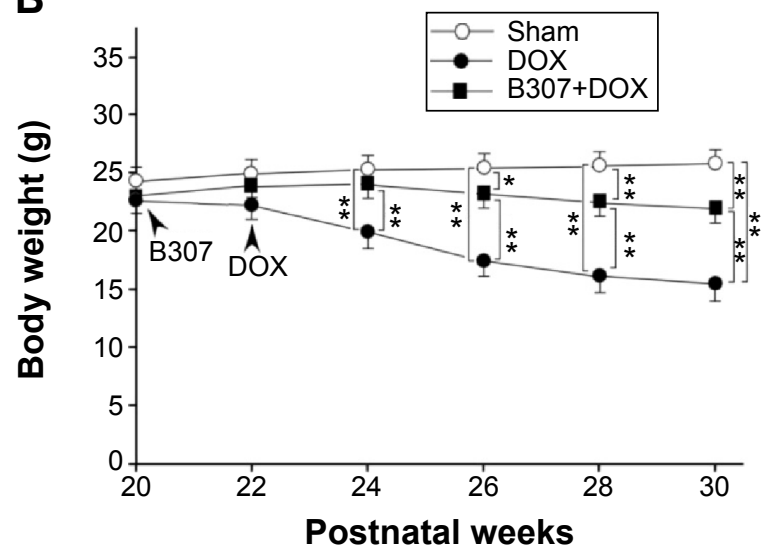

Figure 3 Survival rates and body weight of mice under sham, DOX, and B307+DOX treatments.

Notes: (A) Survival rates of mice began to reduce from DOX treatment and thereafter, while they were unchanged in both mice with sham and B307+DOX treatments. (B) Body weight of the DOX-treated mice was significantly reduced in comparison to those mice under sham treatment from 24 weeks of age and thereafter, whereas body weight of DOX-treated mice under oral B307 treatment was significantly increased as compared to DOX-treated mice from 24 weeks of age and thereafter. Furthermore, body weight of DOX-treated mice under oral B307 treatment was significantly reduced in comparison to those mice under sham treatment from 26 weeks of age and thereafter. The number of mice under sham, DOX, and B307+DOX treatments was 10 for each group. Values are mean \pm SEM ( $* P<0.05$, $* * P<0.01$, two-way ANOVA followed by a Student-Newman-Keuls multiple comparisons posttest).

Abbreviations: DOX, doxorubicin; SEM, standard error of the mean; ANOVA, analysis of variance.

$87.6 \pm 9.2 \mathrm{~cm} / \mathrm{second}, P<0.01$; AV $\max : 42.2 \pm 7.1$ vs $74.5 \pm$ $8.4 \mathrm{~cm} /$ second, $P<0.01$ ), while cardiac dysfunction in the DOX-treated mice had significantly increased under oral B307 treatment (DOX group vs B307+DOX group, HR: $252 \pm 25$ vs $294 \pm 24$ bpm, $P<0.05$; FS: $31.2 \% \pm 5.1 \%$ vs $37.5 \% \pm 5.5 \%$, $P<0.05$; EF: $56.1 \% \pm 8.5 \%$ vs $69.8 \% \pm 8.2 \%, P<0.05$; SV: $37.2 \pm 3.5$ vs $48.9 \pm 3.7 \mu \mathrm{L}, P<0.01 ; \mathrm{CO}: 15.3 \pm 1.4 \mathrm{vs} 18.5 \pm 1.3 \mathrm{~mL} /$ $\min , P<0.01$; MV max: $35.5 \pm 8.3$ vs $76.3 \pm 8.2 \mathrm{~cm} / \mathrm{second}$, $P<0.01$; AV max: $42.2 \pm 7.1$ vs $63.6 .5 \pm 6.7 \mathrm{~cm} / \mathrm{second}$, $P<0.01)$. We also observed that LVFS, EF, SV, CO, MV max, and AV max in the DOX-treated mice under oral B307 treatment were significantly lower than those mice given the sham treatment $(\mathrm{B} 307+\mathrm{DOX}$ group vs sham group, HR: $294 \pm 24$ vs $327 \pm 27$ bpm, $P>0.05$; FS: $37.5 \% \pm 5.5 \%$ vs $50.5 \% \pm 5.4 \%, P<0.01$; EF: $69.8 \% \pm 8.2 \%$ vs $98.5 \% \pm 11.2 \%$, $P<0.05$; SV $: 48.9 \pm 3.7$ vs $55.6 \pm 4.4 \mu \mathrm{L}, P<0.01 ; \mathrm{CO}: 18.5 \pm 1.3$ vs $20.2 \pm 2.1 \mathrm{~mL} / \mathrm{min}, P<0.05$; MV max: $76.3 \pm 8.2$ vs $87.6 \pm 9.2$ $\mathrm{cm} /$ second, $P<0.05$; AV max: $63.6 .5 \pm 6.7$ vs $74.5 \pm 8.4 \mathrm{~cm} /$ second, $P<0.05)$.

\section{Subcutaneous circulation and cardiac expressions of vasodilation-related protein (eNOS) of mice under sham, B307, DOX, and B307+DOX treatment}

Figure 5A shows an example of dorsal imaging of subcutaneous circulation and cardiac expressions of eNOS in the mice at 23-24 weeks of age under sham, B307, DOX, and B307+DOX treatments by using a moorFLPI laser Doppler imager. We quantified and then found that subcutaneous circulation in the mice was significantly enhanced under

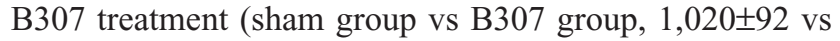
1,445 \pm 95 flux, $P<0.01$ ), but was significantly reduced under DOX treatment (sham group vs DOX group, 1,020 \pm 92 vs $395 \pm 105$ flux, $P<0.01)$. As to the DOX-treated mice, quantified subcutaneous circulation was significantly enhanced under B307 treatment (DOX group vs B307+DOX group, $395 \pm 105$ vs $1,060 \pm 140$ flux, $P<0.01)$.

Figure 5B shows H\&E and IHC staining in the heart tissue of mice at 23-24 weeks of age under sham, B307, DOX, and B307+DOX treatments. As observed from H\&E staining, we found that cardiac fibrosis deposition was obvious in the heart tissue of mice under DOX treatment but was alleviated under oral B307 treatment in the DOX-treated mice. Furthermore, IHC staining illustrated that cardiac expression levels of eNOS in the mice were visibly enhanced under oral B307 treatment but were visibly reduced under DOX treatment. As to the DOXtreated mice, cardiac expression levels of eNOS were visibly enhanced under oral B307 treatment. We quantified eNOS levels in the heart tissue of mice by Western blotting analysis. Our results had shown that eNOS levels in the heart tissue of the mice were significantly enhanced under oral B307 treatment (sham group vs B307 group, $87.5 \% \pm 17.2 \%$ vs $117.6 \% \pm 18.7 \%, P<0.05)$, but were significantly reduced under DOX treatment (sham group vs DOX group, $87.5 \% \pm 17.2 \%$ vs $63.2 \% \pm 19.3 \%, P<0.01$ ). As to the DOX-treated mice, quantified eNOS levels in the heart tissue were significantly enhanced under oral B307 treatment (DOX group vs B307+DOX group, 63.2\% $\pm 19.3 \%$ vs $105.6 \% \pm 20.4 \%, P<0.01)$. 

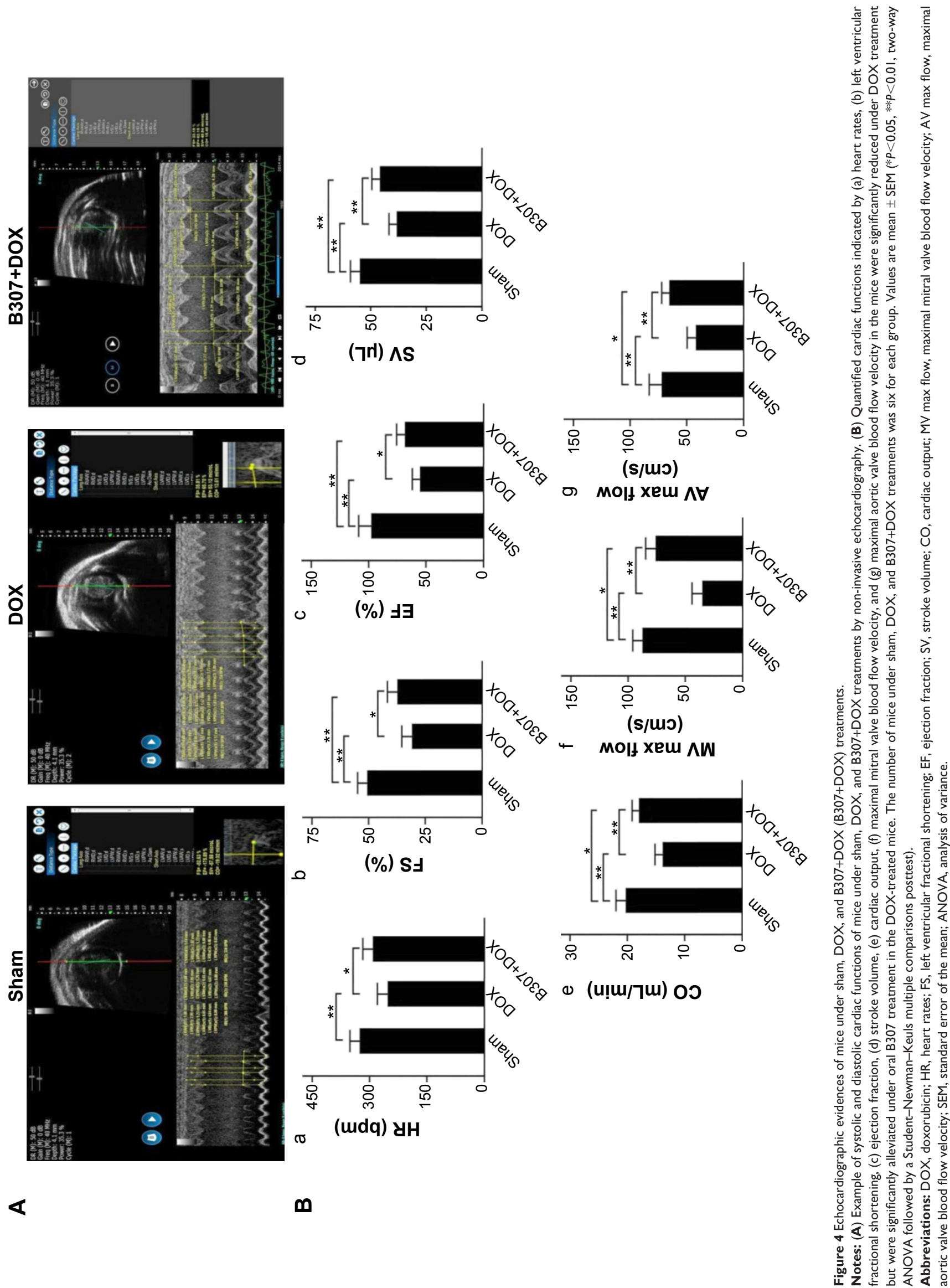

$\varangle$

$\boldsymbol{m}$

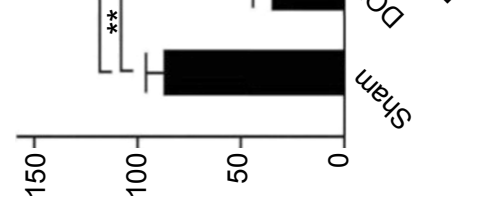


A

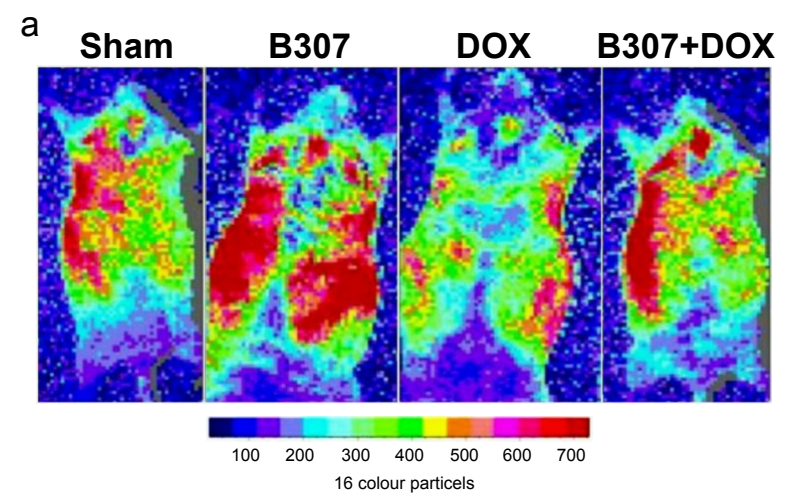

B
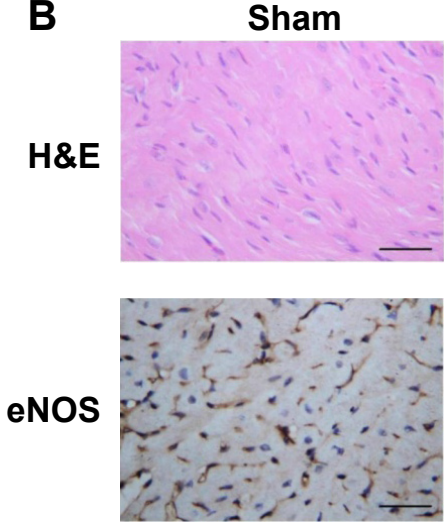

C
B307
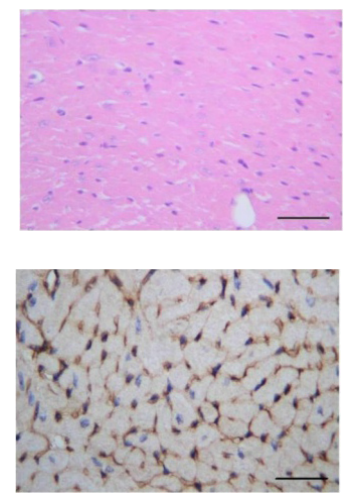

a

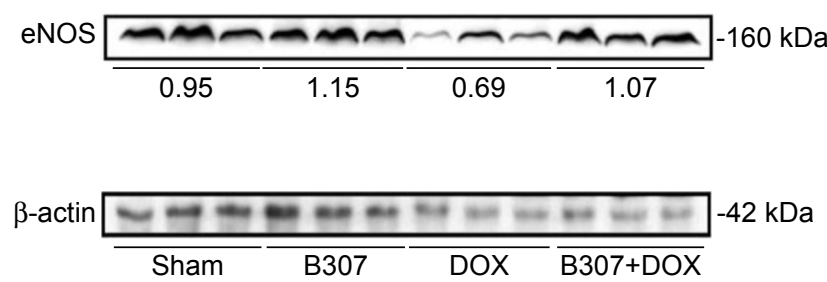

b

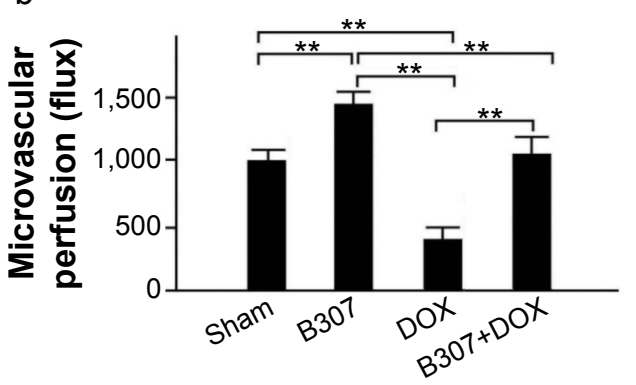

DOX
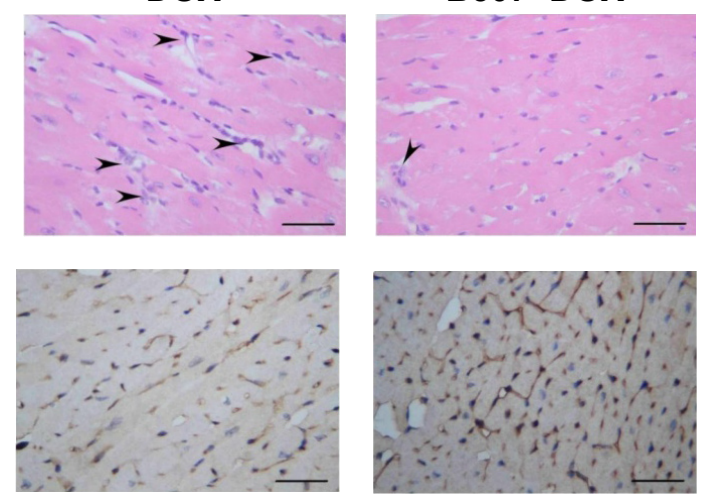

b

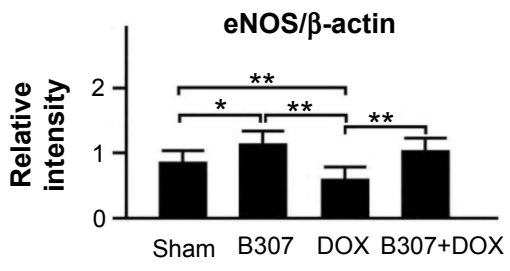

Figure 5 Subcutaneous microcirculation and cardiac expressions of eNOS of mice under sham, B307, DOX, and B307+DOX treatments.

Notes: (A) (a) Dorsal imaging of subcutaneous microcirculation in mice under sham, B307, DOX, and B307+DOX treatments by using moorFLPI laser Doppler imaging and (b) quantified subcutaneous microcirculation of the mice was significantly enhanced under B307 treatment but was significantly reduced under DOX treatment. As to the DOX-treated mice, quantified skin blood flow was significantly enhanced under B307 treatment. (B) H\&E and IHC staining in the heart tissue of the mice under sham, B307, DOX, and B307+DOX treatments. H\&E staining shows cardiac fibrosis deposition (marked by arrows) in the heart tissue of mice under DOX treatment but was alleviated under oral B307 treatment in the DOX-treated mice. IHC staining illustrates that cardiac expression levels of eNOS in the mice were visibly enhanced under oral B307 treatment but were visibly reduced under DOX treatment. As to the DOX-treated mice, cardiac expression levels of eNOS were visibly enhanced under oral B307 treatment. (C) Western blotting analysis shows the following: (a) expression levels of cardiac eNOS under sham, B307, DOX, and B307+DOX treatments and (b) quantified eNOS levels in the heart tissue of the mice were significantly enhanced under oral B307 treatment but were significantly reduced under DOX treatment. As to the DOX-treated mice, quantified eNOS levels in the heart tissue were significantly enhanced under oral B307 treatment. The number of mice under sham, B307, DOX, and B307+DOX treatments was six for each group. Values are mean \pm SEM $(* P<0.05, * * P<0.01$, two-way ANOVA followed by a Student-Newman-Keuls multiple comparisons posttest).

Abbreviations: H\&E, hematoxylin and eosin; DOX, doxorubicin; eNOS, endothelial nitric oxide synthase; kDa, kilodalton; IHC, immunohistochemical; SEM, standard error of the mean; ANOVA, analysis of variance.

\section{Cardiac expressions of oxidative stress (ROS production, SOD2, and 3-NT) in mice under sham, DOX, and B307+DOX treatment}

Figure 6A shows blood ROS production in the mice at 24-25 weeks of age with sham, B307, DOX, and B307+DOX treatments by using a CLD-110 CL detector. We observed that the blood ROS production in the mice was reduced under B307 treatment but was enhanced under DOX treatment. As to the DOX-treated mice, blood ROS production was reduced under B307 treatment. Furthermore, total ROS counts in the mice were significantly reduced under B307 treatment 
A

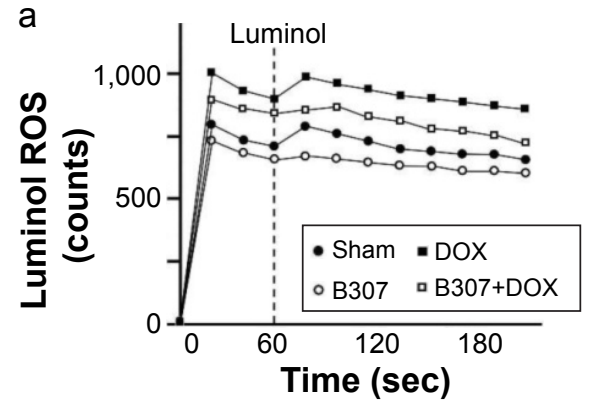

B
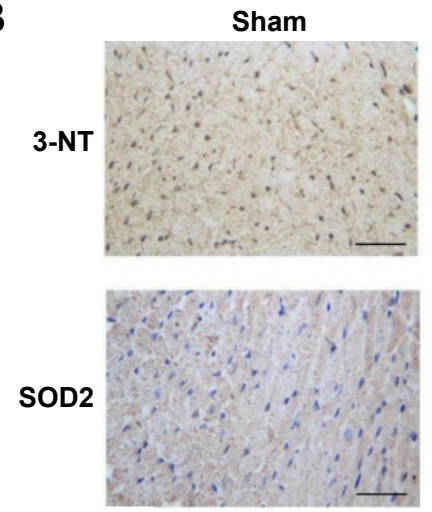

C

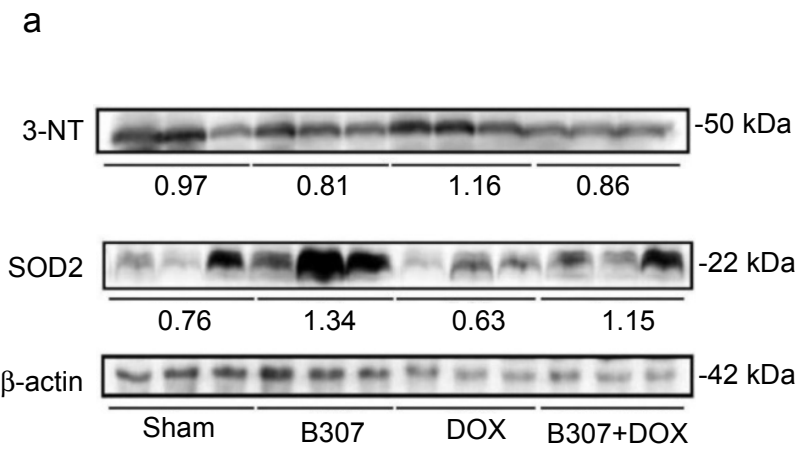

B307
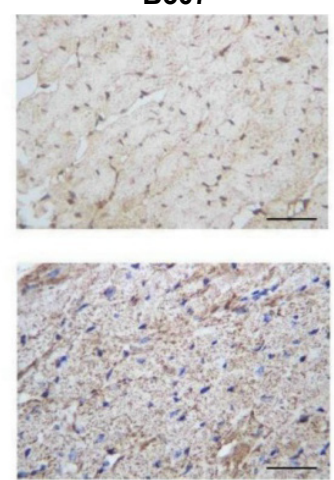
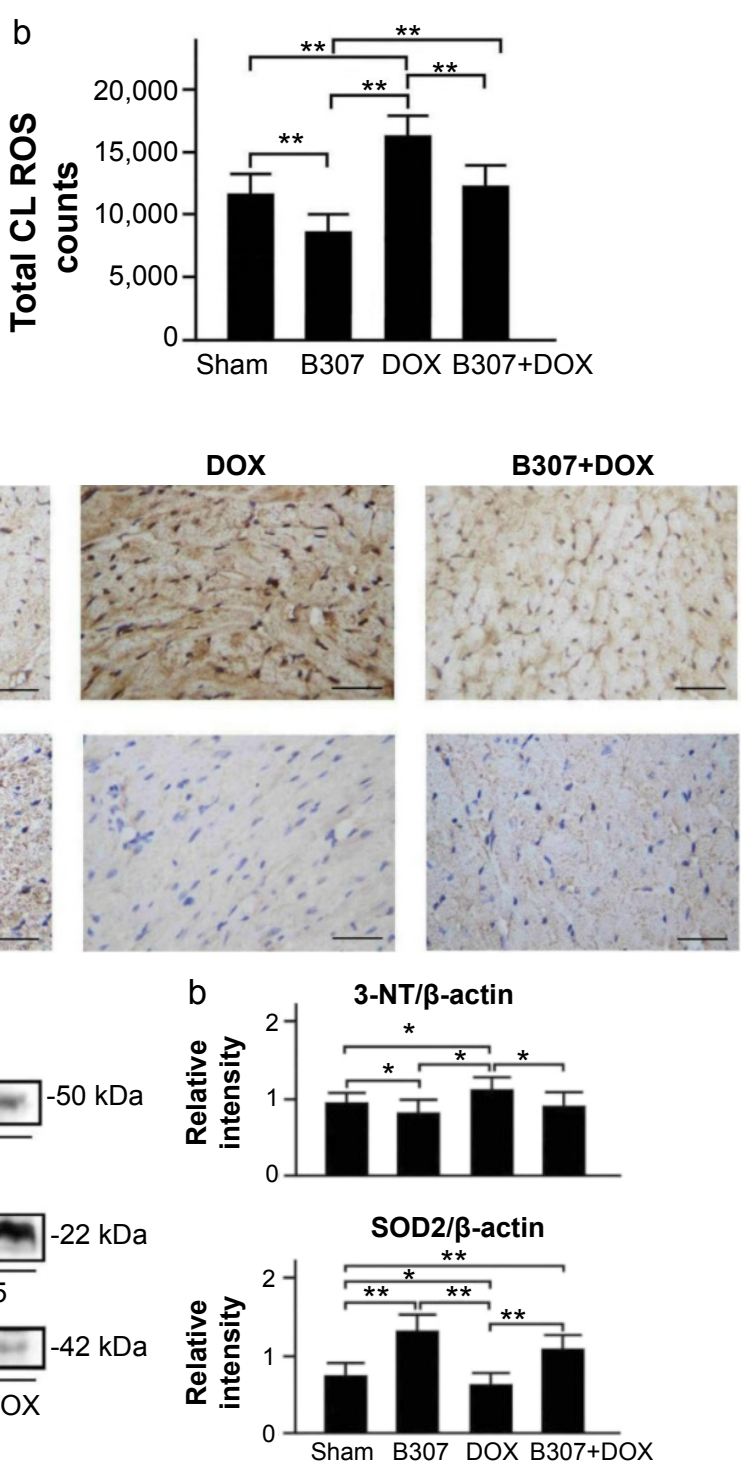

Figure 6 Blood ROS production and cardiac expressions of SOD2, a marker of anti-oxidative stress, and 3-NT, a marker of oxidative stress, in mice under sham, B307, DOX, and B307+DOX treatments.

Notes: (A) Blood ROS production of mice under sham, B307, DOX, and B307+DOX treatments by using a CLD-II0 CL detector, (a) blood ROS production in the mice was reduced under B307 treatment but was enhanced under DOX treatment. As to the DOX-treated mice, blood ROS production was reduced under B307 treatment, and (b) total ROS counts in the mice were significantly reduced under B307 treatment but were significantly enhanced under DOX treatment. As to the DOX-treated mice, total ROS counts were significantly reduced under B307 treatment. (B) IHC staining illustrates that cardiac expression levels of SOD2 in the mice were visibly enhanced under oral B307 treatment but were visibly reduced by DOX treatment. As to the DOX-treated mice, cardiac expression levels of SOD2 were visibly enhanced under oral B307 treatment. On the other hand, cardiac expression levels of 3-NT of mice were visibly reduced under oral B307 treatment but were visibly enhanced under DOX treatment. As to the DOX-treated mice, cardiac expression levels of 3-NT were visibly reduced under oral B307 treatment. (C) Western blotting analysis shows the following: (a) expression levels of cardiac SOD2 and 3-NT under sham, B307, DOX, and B307+DOX treatments and (b) quantified SOD2 levels in the heart tissue of the mice were significantly enhanced under oral B307 treatment but were significantly reduced under DOX treatment. As to the DOX-treated mice, quantified SOD2 levels in heart tissue were significantly enhanced under oral B307 treatment. On other hand, quantified 3-NT levels in the heart tissue of mice were significantly reduced under oral B307 treatment, but were significantly enhanced under DOX treatment. As to the DOX-treated mice, quantified 3-NT levels in the heart tissue were significantly reduced under oral B307 treatment. The number of mice under sham, B307, DOX, and B307+DOX treatments was six for each group. Values are mean $\pm S E M(* P<0.05$, $* * P<0.0$ I, two-way ANOVA followed by a Student-Newman-Keuls multiple comparisons posttest).

Abbreviations: ROS, reactive oxygen species; CL, luminol chemiluminescence; DOX, doxorubicin; 3-NT, neurotrophin-3; SOD2, superoxide dismutase 2; kDa, kilodalton; $\mathrm{IHC}$, immunohistochemical; SEM, standard error of the mean; ANOVA, analysis of variance.

(sham group vs B307 group, 11,715 $\pm 1,635$ vs $8,733 \pm 1,349$ counts, $P<0.01$ ), but were significantly enhanced under DOX treatment (sham group vs DOX group, 11,715 $\pm 1,635$ vs $16,401 \pm 1,623$ counts, $P<0.01$ ). As to the DOX-treated mice, total ROS counts were significantly reduced under B307 treatment (DOX group vs B307+DOX group, 16,401 $\pm 1,623$ vs $12,212 \pm 1,704$ counts, $P<0.01$ ).

Figure 6B shows IHC staining cardiac expression levels of SOD2 in the mice at 24-25 weeks of age were visibly enhanced under oral B307 treatment but were visibly reduced 
under DOX treatment. As to the DOX-treated mice, cardiac expression levels of SOD2 were visibly enhanced under oral B307 treatment. On the other hand, cardiac expression levels of 3-NT in the mice were visibly lowered under oral B307 treatment but were visibly enhanced under DOX treatment. As to the DOX-treated mice, cardiac expression levels of 3-NT were visibly reduced under oral B307 treatment. Figure 6C shows that quantified SOD2 levels in the heart tissue of the mice were significantly enhanced under oral B307 treatment (sham group vs B307 group, $77.5 \% \pm 14.2 \%$ vs $129.6 \% \pm 15.7 \%, P<0.01$ ), but were significantly reduced under DOX treatment (sham group vs DOX group, $77.5 \% \pm 14.2 \%$ vs $63.4 \% \pm 14.4 \%, P<0.05$ ). As to the DOX-treated mice, quantified SOD2 levels in the heart tissue were significantly enhanced under oral B307 treatment (DOX group vs B307+DOX group, $63.4 \% \pm 14.4 \%$ vs $108 \% \pm 13.3 \%, P<0.01)$. On the other hand, quantified 3-NT levels in heart tissue of the mice were significantly reduced under oral B307 treatment (sham group vs B307 group, $94.6 \% \pm 15.1 \%$ vs $84.6 \% \pm 14.2 \%, P<0.05$ ), but were significantly enhanced under DOX treatment (sham group vs DOX group, $94.6 \% \pm 15.1 \%$ vs $112.5 \% \pm 16.3 \%, P<0.05$ ). As to the DOX-treated mice, quantified 3-NT levels in heart tissue were significantly reduced under oral B307 treatment (DOX group vs B307+DOX group, $112.5 \% \pm 16.3 \%$ vs $108.2 \% \pm 13.4 \%, P<0.05)$.

\section{Cardiac expressions of inflammation (NF- $\kappa B, T N F-\alpha$ ) in mice under sham, DOX, and B307+DOX treatment}

Figure 7A shows IHC staining that cardiac expression levels of NF- $\kappa$ B and TNF- $\alpha$ of mice at the age of 24-25 weeks were visibly reduced under oral B307 treatment, but were visibly enhanced under DOX treatment. As to the DOX-treated mice, cardiac expression levels of NF- $\kappa$ B and TNF- $\alpha$ were visibly reduced under oral B307 treatment. Figure 7B shows that quantified TNF- $\alpha, \mathrm{NF}-\kappa \mathrm{B}$ precursor, and $\mathrm{p} 50-\mathrm{NF}-\kappa \mathrm{B}$ levels in heart tissue of the mice were not significant under oral B307 treatment (sham group vs B307 group, TNF- $\alpha$ : $83.3 \% \pm 21.6 \%$ vs $76.5 \% \pm 18.5 \%, P>0.05$; NF- $\kappa$ B precursor: $69.2 \% \pm 25.2 \%$ vs $63.7 \% \pm 19.5 \%$; p50-NF- $\kappa B: 108.6 \% \pm 25.2 \%$ vs $96.1 \% \pm 18.5 \%, P>0.05)$, but were significantly enhanced under DOX treatment (sham group vs DOX group, TNF- $\alpha$ : $83.3 \% \pm 21.6 \%$ vs $181.2 \% \pm 25.3 \%, P<0.01$; NF- $\kappa$ B precursor: $69.2 \% \pm 25.2 \%$ vs $132.1 \% \pm 23.4 \%, P<0.01$; $550-\mathrm{NF}-\kappa \mathrm{B}$ : $108.6 \% \pm 25.2 \%$ vs $179.6 \% \pm 25.3 \%, P<0.01)$. As to the DOXtreated mice, quantified TNF- $\alpha$ and NF- $\kappa B$ of 50 and $65 \mathrm{kDa}$ levels in heart tissue, respectively, were significantly reduced under oral B307 treatment (DOX group vs B307+DOX group, TNF- $\alpha$ : $181.2 \% \pm 25.3 \%$ vs $92.3 \% \pm 22.3 \%, P<0.01 ;$ NF- $\kappa \mathrm{B}$ precursor: $132.1 \% \pm 23.4 \%$ vs $91.1 \% \pm 24.4 \%, P<0.01$; p50NF-кB: $179.6 \% \pm 25.3 \%$ vs $91.6 \% \pm 22.3 \%, P<0.01)$.

\section{Cardiac expressions of apoptosis (Bcl-2, Bax, Cyto-C, calpain, caspase 12, caspase 9, and caspase 3) of mice under sham, DOX, and B307+DOX treatment}

Figure 8A shows IHC staining cardiac expression levels of Bcl-2 in the mice at 24-25 weeks of age were visibly enhanced under oral B307 treatment but were visibly reduced under DOX treatment. As to the DOX-treated mice, cardiac expression levels of Bcl-2 were visibly enhanced under oral B307 treatment. On the other hand, cardiac expression levels of Bax and Cyto-C in the mice were visibly reduced under oral B307 treatment but were visibly enhanced under DOX treatment. As to the DOX-treated mice, cardiac expression levels of Bax and Cyto-C were visibly reduced under oral B307 treatment. Figure $8 \mathrm{~B}$ shows the quantified ratio of $\mathrm{Bcl}-2 / \mathrm{Bax}$ in the heart tissue of the mice was significantly enhanced under oral B307 treatment (sham group vs B307 group, $P<0.01$ ) but was not significant under DOX treatment (sham group vs DOX group, $P>0.05$ ). As to the DOX-treated mice, quantified SOD2 levels in the heart tissue were significantly enhanced under oral B307 treatment (DOX group vs B307+DOX group, $P<0.01)$. On the other hand, quantified Cyto-C levels in the heart tissue of the mice were significantly reduced under oral B307 treatment (sham group vs B307 group, $P<0.05)$ but were significantly enhanced under DOX treatment (sham group vs DOX group, $P<0.01-0.05$ ). As to the DOX-treated mice, quantified Cyto-C levels in the heart tissue were significantly reduced under oral B307 treatment (DOX group vs B307+DOX group, $P<0.01$ ).

Figure 9A shows that IHC staining cardiac expression levels of calpain and caspase 12 in the mice at 24-25 weeks of age were slightly reduced under oral B307 treatment but were visibly enhanced under DOX treatment. As to the DOX-treated mice, cardiac expression levels of calpain and caspase 12 were visibly decreased under oral B307 treatment. Figure 9B shows that quantified calpain, pro-caspase 12, and c-caspase 12 levels in the heart tissue of the mice were not significant under oral B307 treatment (sham group vs B307 group, $P>0.05$ ) but were significantly enhanced under DOX treatment (sham group vs DOX group, $P<0.01$ ). As to the DOX-treated mice, quantified calpain, pro-caspase 12 , and c-caspase 12 levels in the heart tissue were significantly reduced under oral B307 treatment (DOX group vs 
A Sham

NF-kB

NF- $\alpha$

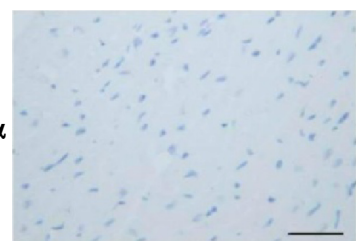

B307
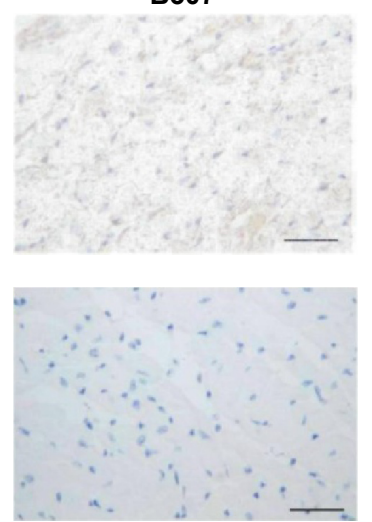

DOX

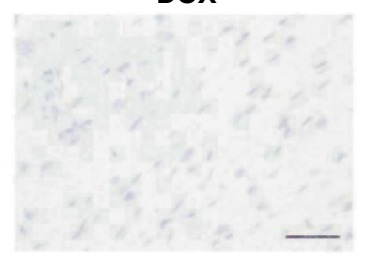

B307+DOX
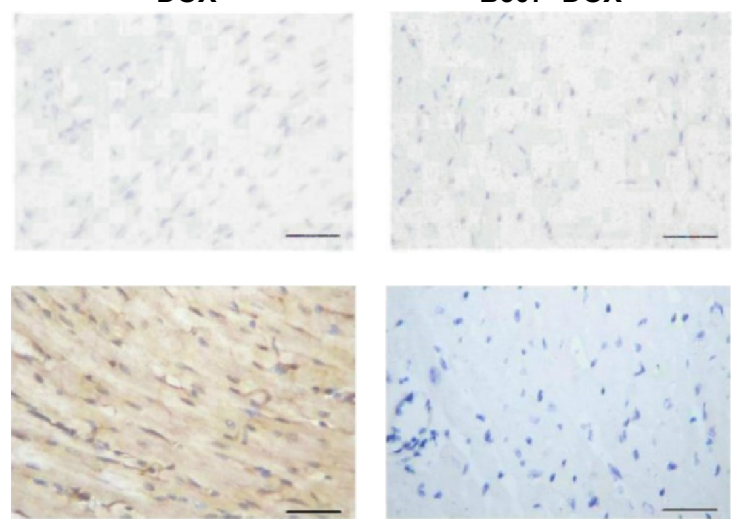

B

a TNF- $\alpha$

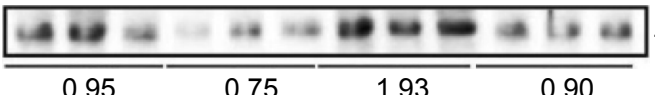

$\beta$-actin

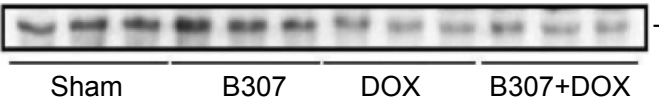
$-28 \mathrm{kDa}$ $-42 \mathrm{kDa}$

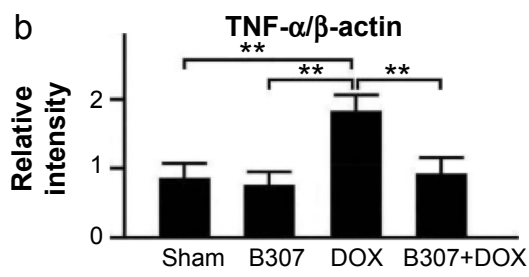

C a
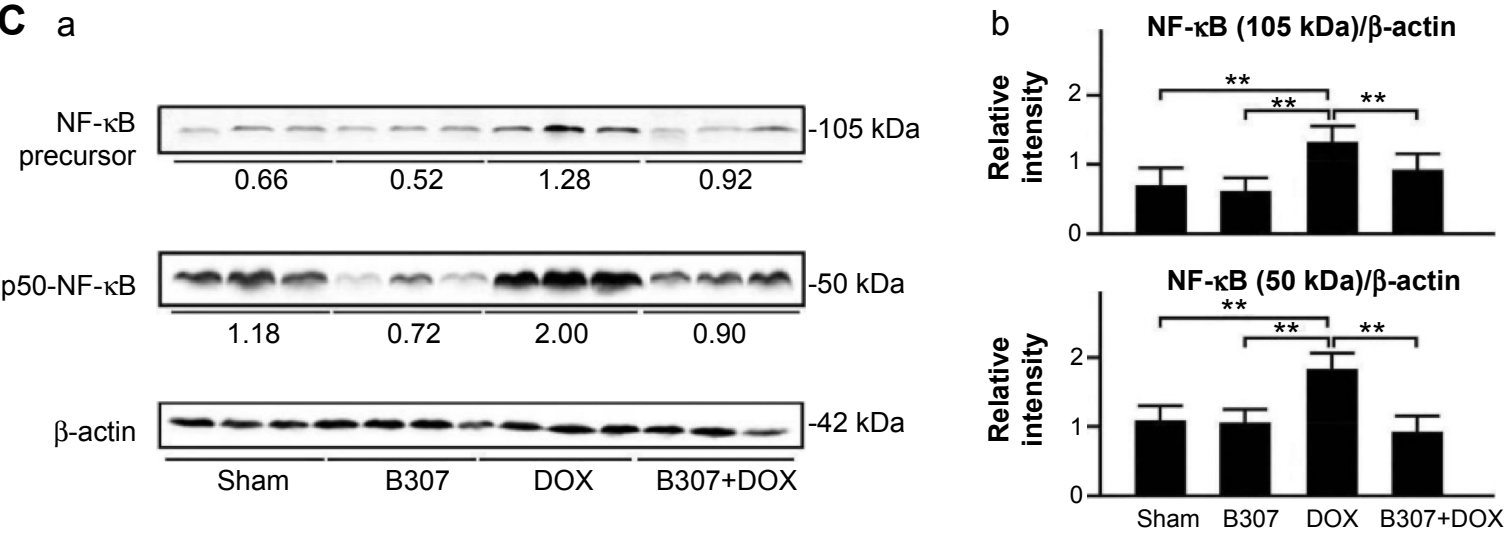

Figure 7 Cardiac levels of TNF- $\alpha$, NFKBI (p50 and its precursor, pl05), markers of inflammation, in mice under sham, B307, DOX, and B307+DOX treatments. Notes: (A) IHC staining illustrates that cardiac expression levels of NF- $\mathrm{KB}$ and TNF- $\alpha$ in the mice were visibly enhanced under DOX treatment. As to the DOX-treated mice, cardiac expression levels of NF- $\mathrm{KB}$ and TNF- $\alpha$ were visibly reduced under oral B307 treatment. (B) Western blotting analysis shows the following: (a) expression levels of cardiac TNF- $\alpha$ under sham, B307, DOX, and B307+DOX treatments and (b) quantified TNF- $\alpha$ levels in the heart tissue of mice were significantly increased under DOX treatment. As to the DOX-treated mice, quantified TNF- $\alpha$ levels in the heart tissue were significantly decreased under oral B307 treatment. (C) Western blotting analysis shows the following: (a) expression levels of cardiac NFKBI (p50 and its precursor, pl05) under sham, B307, DOX, and B307+DOX treatments, and (b) quantified levels of NFKBI (p50 and its precursor, pl05) in the heart tissue of the mice were significantly increased under DOX treatment. As to the DOX-treated mice, quantified levels of NFKBI (p50 and its precursor, pl05) in the heart tissue were significantly decreased under oral B307 treatment. The number of mice under sham, B307, DOX, and B307+DOX treatments was six for each group. Values are mean \pm SEM ( $* * P<0.01$, two-way ANOVA followed by a Student-Newman-Keuls multiple comparisons posttest).

Abbreviations: NF- $\mathrm{KB}$, nuclear factor- $\mathrm{KB}$; TNF- $\alpha$, tumor necrosis factor alpha; DOX, doxorubicin; kDa, kilodalton; IHC, immunohistochemical; SEM, standard error of the mean; ANOVA, analysis of variance.

B307+DOX group, $P<0.01)$. Similarly, Figure 10A shows that IHC staining cardiac expression levels of caspase 9 and caspase 3 in the mice were slightly reduced under oral B307 treatment but were visibly enhanced under DOX treatment. As to the DOX-treated mice, cardiac expressions of caspase 9 and caspase 3 were visibly reduced under oral B307 treatment. Figure 10B shows that only quantified caspase 3 levels in the heart tissue of the mice were significantly reduced under oral B307 treatment (sham group vs B307 group, $P<0.05$ ) but quantified pro-caspase 9, c-caspase 9, pro-caspase 3 , and c-caspase 3 levels were significantly enhanced under DOX treatment (sham group vs DOX group, $P<0.01)$. As to the DOX-treated mice, quantified pro-caspase 9 , c-caspase 9, pro-caspase 3, and c-caspase 3 levels in the heart tissue were significantly reduced under oral B307 treatment (DOX group vs B307+DOX group, $P<0.01$ ). 
A
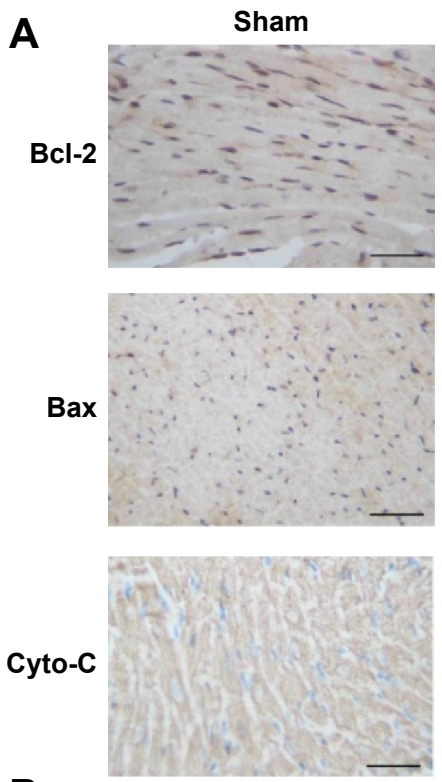

B

a
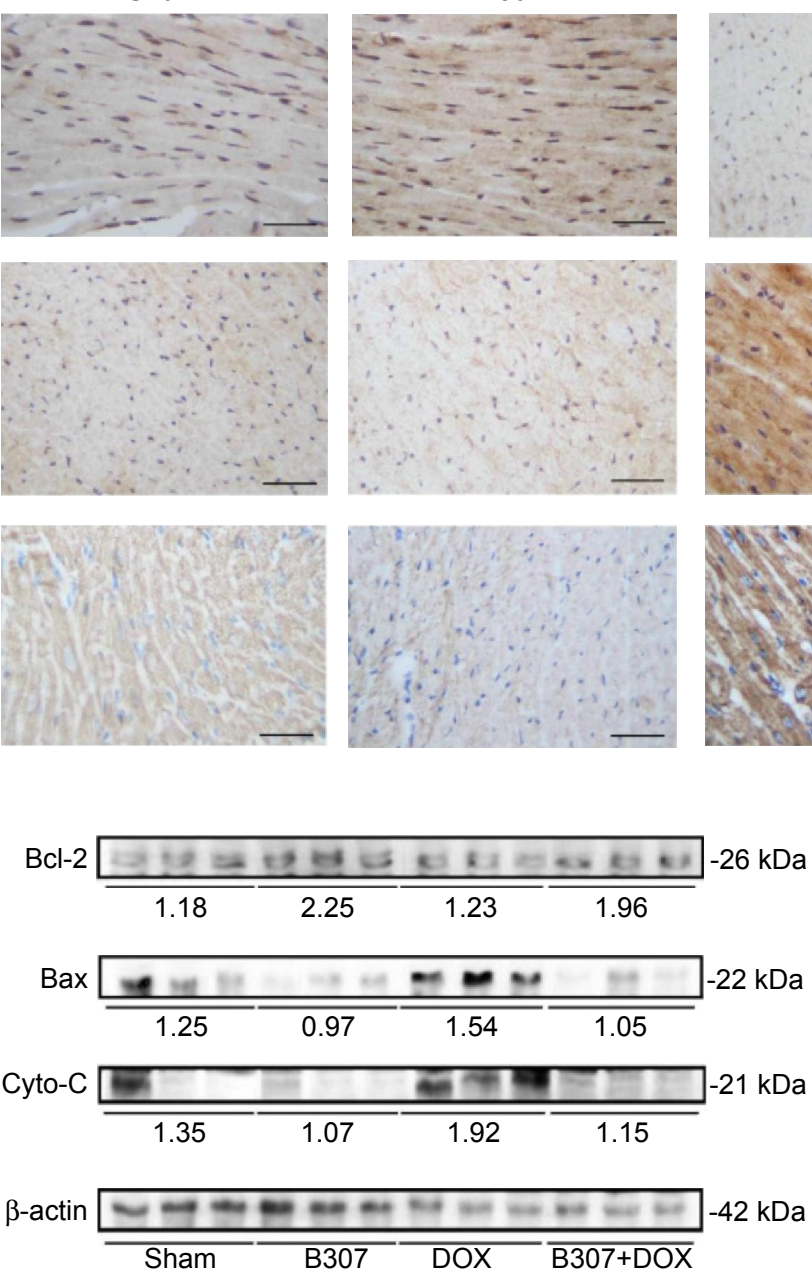

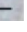

(1)
DOX
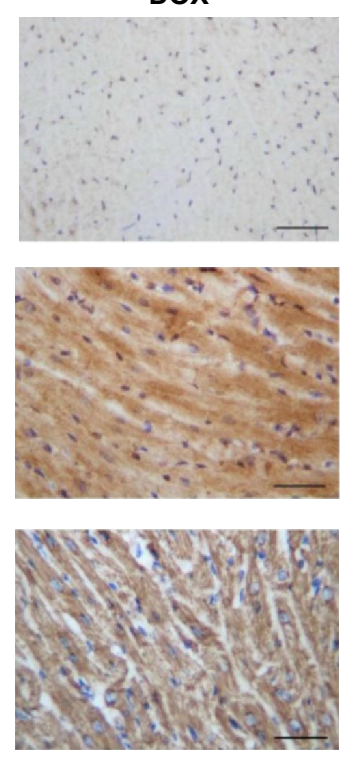

b
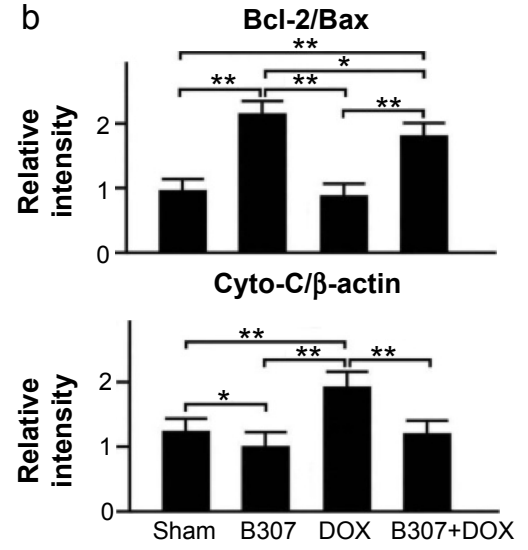

Figure 8 Cardiac levels of Bcl-2, a marker of anti-apoptosis, and Bax and Cyto-C, two markers of apoptosis, in mice under sham, B307, DOX, and B307+DOX treatments.

Notes: (A) IHC staining illustrates that cardiac expression levels of Bcl-2 in the mice were visibly enhanced under oral B307 treatment but were visibly reduced under DOX treatment. As to the DOX-treated mice, cardiac expression levels of $\mathrm{Bcl}-2$ were visibly enhanced under oral B307 treatment. On the other hand, cardiac expression levels of Bax and Cyto-C in the mice were visibly reduced under oral B307 treatment but were visibly enhanced under DOX treatment. As to the DOX-treated mice, cardiac expression levels of Bax and Cyto-C were visibly reduced under oral B307 treatment. (B) Western blotting analysis shows the following: (a) expression levels of cardiac Bcl-2, Bax, and Cyto-C under sham, B307, DOX, and B307+DOX treatments and (b) the quantified ratio of Bcl-2/Bax in the heart tissue of the mice was significantly increased under oral B307 treatment but were significantly reduced under DOX treatment. As to the DOX-treated mice, the quantified ratio of Bcl-2/Bax in the heart tissue was significantly increased under oral B307 treatment. On the other hand, quantified Cyto-C levels in the heart tissue of the mice were significantly decreased under oral B307 treatment but were significantly enhanced under DOX treatment. As to the DOX-treated mice, quantified Cyto-C levels in the heart tissue were significantly reduced under oral B307 treatment. The number of mice under sham, B307, DOX, and B307+DOX treatments was six for each group. Values are mean $\pm S E M(* P<0.05$, ** $P<0.0$, two-way ANOVA followed by a Student-Newman-Keuls multiple comparisons posttest).

Abbreviations: Bcl-2, B-cell lymphoma 2; Bax, BCL2-associated X protein; Cyto-C, cytochrome C; DOX, doxorubicin; kDa, kilodalton; IHC, immunohistochemical; SEM, standard error of the mean; ANOVA, analysis of variance.

\section{Discussion}

In this study, we evaluated the potential of the herbal formula B307 in ameliorating DOX-induced acute cardiotoxicity in mice and elucidated the underlying molecular mechanisms. Alleviative effects of the herbal formula B307 for DOXinduced cardiotoxicity were assessed by examining cardiac functions via using non-invasive echocardiography, subcutaneous circulation, and moorFLPI laser Doppler imaging. IHC and Western blotting analysis were used to examine the expression levels of vasodilation, oxidative stress, inflammation, and the formation of apoptosis-related proteins. As can be seen from charts of LC/MS chromatography in Figure 1, we observed that the herbal formula B307 mainly contains ingredients of Ginsenosides Rb1 from $P$. ginseng, rosmarinic acid, salvianolic acid B, tanshinone IIA from Salvia miltiorrhiza, schizandrin and gomisin A from Schisandra chinensis, and methylophiopogonanone B from Liriope spicata. Ginseng has been widely used as a traditional herbal medicine that 
A
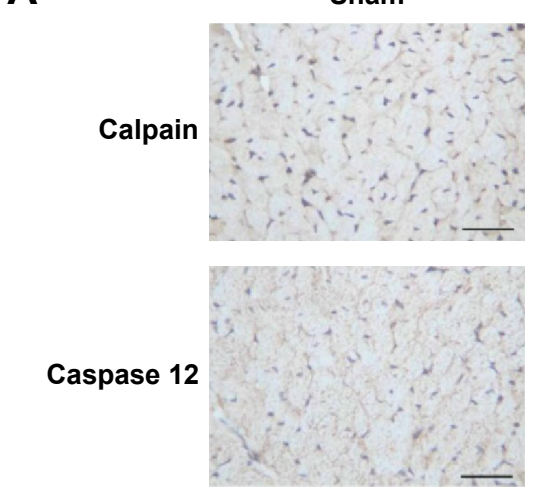

B

a

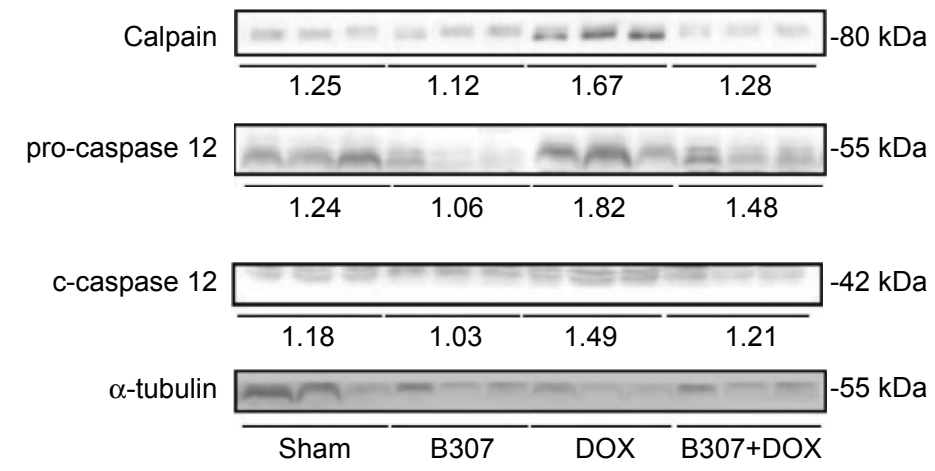

B307
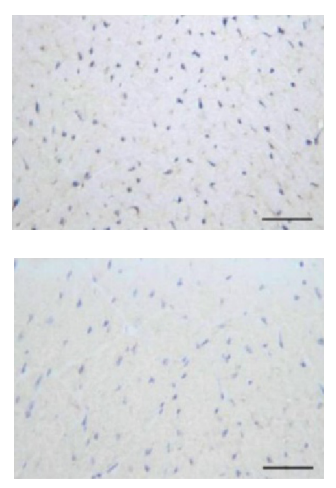

DOX
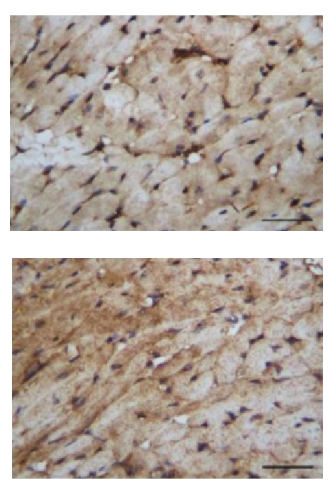

B307+DOX
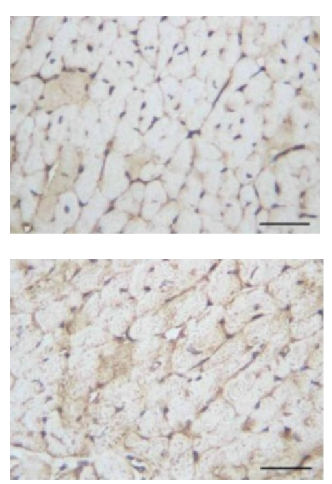

b
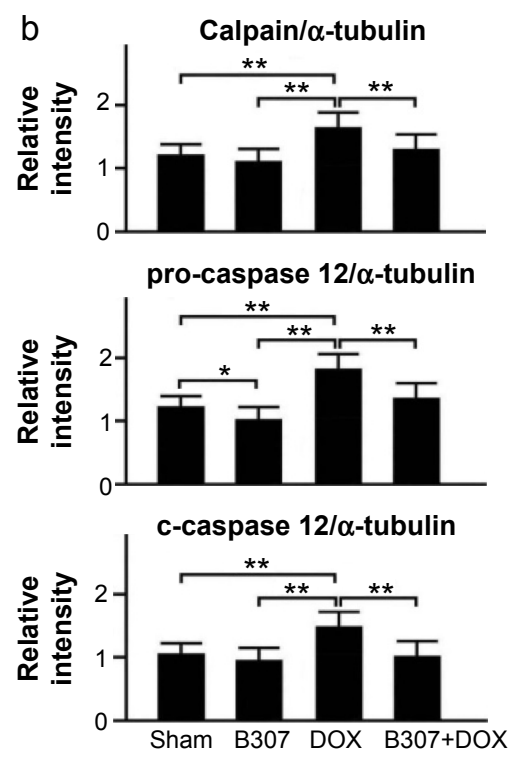

Figure 9 Cardiac levels of calpain, pro-caspase 12, c-caspase I2, ER stress-related apoptotic inducers, in mice under sham, B307, DOX, and B307+DOX treatments. Notes: (A) IHC staining illustrates that cardiac expression levels of calpain and caspase 12 in the mice were visibly reduced under B307 treatment but were visibly enhanced under DOX treatment. As to the DOX-treated mice, cardiac expression levels of calpain, pro-caspase 12 (55 kDa) and c-caspase 12 (42 kDa) were visibly reduced under oral B307 treatment. (B) Western blotting analysis shows the following: (a) expression levels of cardiac calpain, pro-caspase I2, and c-caspase I2 under sham, B307, DOX, and B307+DOX treatments and (b) quantified levels of calpain, pro-caspase 12, and c-caspase 12 in the heart tissue of the mice were significantly increased under DOX treatment. As to the DOX-treated mice, quantified calpain, pro-caspase I2, and c-caspase 12 levels in the heart tissue were significantly decreased under oral B307 treatment. The number of mice under sham, B307, DOX, and B307+DOX treatments was six for each group. Values are mean \pm SEM (*P $<0.05$, **P $<0.0 \mathrm{I}$, two-way ANOVA followed by a Student-Newman-Keuls multiple comparisons posttest).

Abbreviations: ER, endoplasmic reticulum; DOX, doxorubicin; kDa, kilodalton; IHC, immunohistochemical; SEM, standard error of the mean; ANOVA, analysis of variance.

exhibits multifunctional activities such as anti-excitotoxicity, antioxidant, anti-inflammatory, and altered expression of neurotrophic factors. ${ }^{9-14}$ However, Danshen has been widely used to treat heart disease and ameliorate an atherosclerosis effect in humans and rodents. ${ }^{15-17}$ Furthermore, we found that the main component of the herbal formula B307 is Shengmai San. Shengmai San has been widely used in the People's Republic of China that is administered to patients who have heart attack and congestive heart failure. Thus, we selected herbal formula B307 to treat cardiac failure of DOX-treated mice.

Before evaluating the potential of the herbal formula B307 in ameliorating DOX-induced acute cardiotoxicity, we confirmed the safety of the herbal formula B307 via the in vitro experiment. To this end, we tested the concentration of $50 \%$ inhibition $\left(\mathrm{IC}_{50}\right)$ of the herbal formula $\mathrm{B} 307$ by examining cell viability in Huh7 cancer cells under B307 treatment. We found that cell viability in Huh7 cancer cells was not affected under B307 treatment at 10-160 $\mu \mathrm{g} / \mathrm{mL}$ (Figure 2A, $P>0.01)$. In the next step, we confirmed if the herbal formula B307 reduced the chemotherapeutic effect of DOX in cancer cells. In order to do this, we further measured cell viability in Huh7 cancer cells under treatments of DOX and DOX+B307. Our data had shown that cell viability in Huh7 cancer cells was significantly reduced by approximately $50 \%-60 \%$ under DOX treatment at $10-30 \mu \mathrm{g} / \mathrm{mL}$ (Figure $1 \mathrm{C}$, $P<0.01)$. However, there was no significant difference 
A

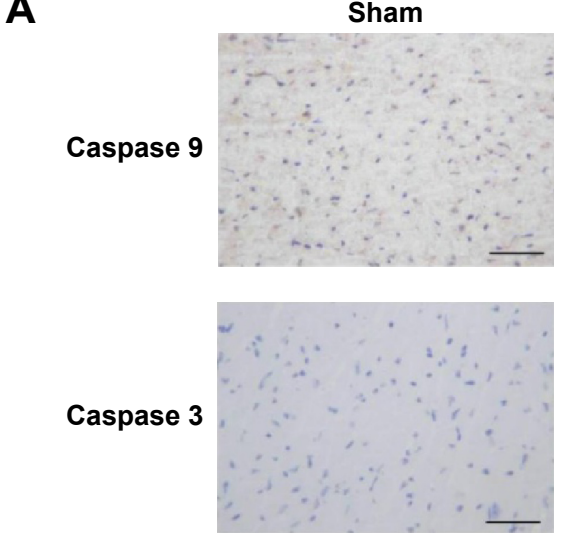

B

pro-caspase 9

a

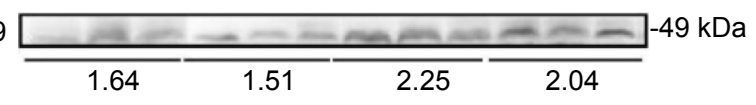

c-caspase 9

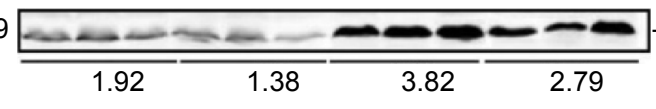

$-39 \mathrm{kDa}$

$\alpha$-tubulin

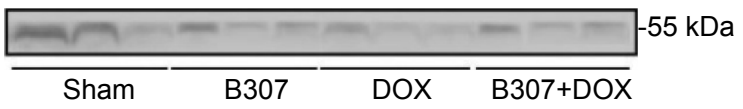

C

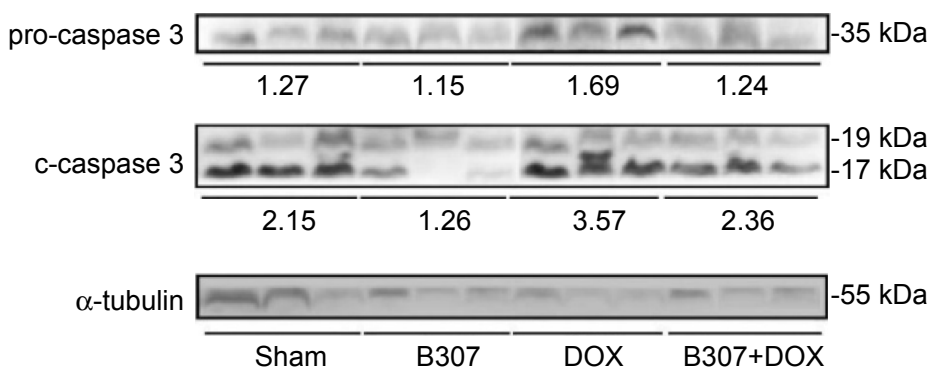

DOX
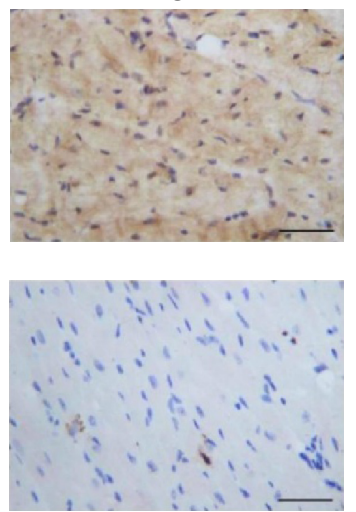

b
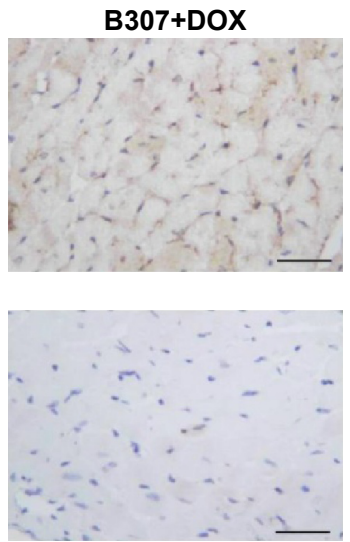

pro-caspase $9 / \alpha$-tubulin

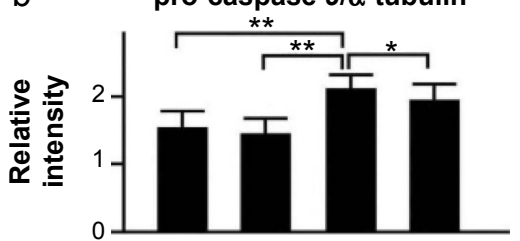

C-caspase $9 / \alpha$-tubulin

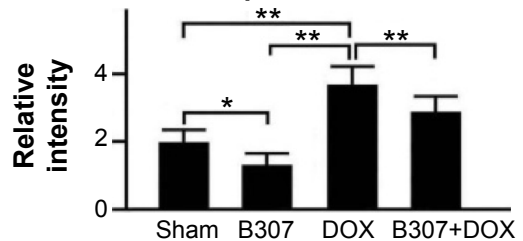

b

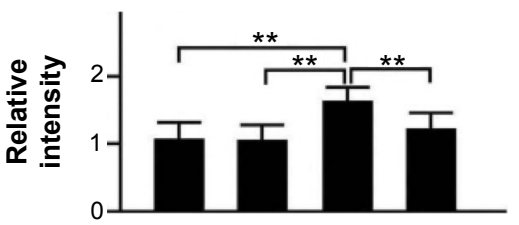

c-caspase $3 / \alpha$-tubulin

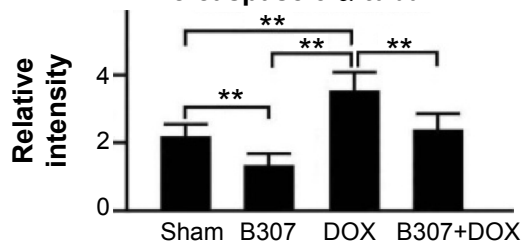

Figure 10 Cardiac levels of pro-caspase 9, c-caspase 9, pro-caspase 3, and c-caspase 3 in mice under sham, B307, DOX, and B307+DOX treatments.

Notes: (A) IHC staining illustrates that cardiac expression levels of caspase 9 and caspase 3 in the mice were visibly reduced under B307 treatment but were visibly enhanced under DOX treatment. As to the DOX-treated mice, cardiac expression levels of calpain and caspase 12 were visibly reduced under oral B307 treatment. (B, C) (a) Western blotting analysis shows the following: (a) expression levels of cardiac pro-caspase 9 (49 kDa), c-caspase 9 (39 kDa), pro-caspase 3 ( $35 \mathrm{kDa})$, and c-caspase 3 (17 and $19 \mathrm{kDa}$ ) under sham, B307, DOX, and B307+DOX treatments, and (b) quantified levels of pro-caspase 9, c-caspase 9, pro-caspase 3, and c-caspase 3 in the heart tissue of the mice were significantly increased under DOX treatment. As to the DOX-treated mice, quantified levels of pro-caspase 9, c-caspase 9, pro-caspase 3, and c-caspase 3 in the heart tissue were significantly decreased under oral B307 treatment. The number of mice under sham, B307, DOX, and B307+DOX treatments was six for each group. Values are mean \pm SEM $(* P<0.05, * * P<0.0$ l, two-way ANOVA followed by a Student-Newman-Keuls multiple comparisons posttest).

Abbreviations: DOX, doxorubicin; $\mathrm{kDa}$, kilodalton; IHC, immunohistochemical; SEM, standard error of the mean; ANOVA, analysis of variance.

between cell viability in Huh7 cancer cells under DOX only and DOX+B307 treatment (Figure 2B, $P>0.05$ ).

DOX treatment has caused marked myocardial dysfunction such as myofibrillar loss, cytoplasmic vacuole formation, inflammatory cell infiltration, hemorrhage, and interstitial edema as has been reported in the previous study. ${ }^{21}$ Evidence from our study had shown that overall survival rate and body weight of the mice were obviously reduced under DOX treatment (Figure 3). Yet, cardiac function and blood circulation in the mice were obviously improved under DOX treatment 
(Figures 4-6). Oxidative stress has been considered a major contributor in DOX-induced cardiac dysfunction. ${ }^{22}$ DOX treatment increases cardiac muscle susceptibility to ROS by reducing activities of antioxidant enzymes such as SOD2. ${ }^{23}$ We observed that blood ROS production was enhanced, while expression of SOD2 was reduced in mice under DOX treatment (Figure 6). Like the previous study, we have observed that DOX treatment induced oxidative damage in the heart tissue by enhancing cardiac expressions of $3-\mathrm{NT} .{ }^{24}$ In this study, we observed that DOX treatment elicits inflammatory effects in the heart tissue by enhancing the expression of TNF- $\alpha$ and NFKB1 (p50 and its precursor, p105) (Figure 7). TNF- $\alpha$ and NFKB1 (p50 and its precursor, p105) are involved in the immune responses and inflammatory responses. ${ }^{25}$ Furthermore, DOX treatment enhances the production of TNF- $\alpha$ and NFKB1 (p50 and its precursor, p105) that has been reported in the previous study. ${ }^{26}$ Besides, previous evidence implicated that DOX-induced apoptotic death of cardiac myocytes is the most direct cause of DOX cardiotoxicity. ${ }^{27}$ Accumulative evidence indicates that cellular apoptosis or programmed cell death may play a critical role in the pathogenesis of DOX-induced cardiotoxicity. ${ }^{28}$ Our results reveal that cardiac expressions of Bcl-2 were suppressed, but those of Bax, calpain, caspase 12, caspase 9, and caspase 3 were enhanced in mice under DOX treatment (Figures 8-10). Apoptosis is the process of programmed cell death that is regulated by a series of regulating proteins. Among these regulating proteins, the $\mathrm{Bcl}-2$ family of proteins are major regulators of apoptosis. ${ }^{29}$ This Bcl-2 family includes proapoptotic proteins, such as Bax, and anti-apoptotic proteins, such as Bcl-2. Cell survival in the early phases of an apoptotic cascade mainly depends on the balance between the pro- and anti-apoptotic proteins of the Bcl-2 family. ${ }^{30}$ Our results had shown that DOX treatment has profound effects on the Bcl-2 family proteins. It downregulated $\mathrm{Bcl}-2$ expression but upregulated Bax expression. Consequently, the ratio of the antiapoptotic Bcl-2 to pro-apoptotic Bax was significantly reduced under DOX treatment (Figure 8). Next, we found that both expressions of calpain and caspase 12 in the heart tissue of the mice were enhanced under DOX treatment (Figure 9). Calpain and caspase are two key markers of endoplasmic reticulum (ER) stress-related apoptosis in cardiac dysfunction. ${ }^{31}$ Similarly, we found that DOX treatment induced marked apoptosis of cardiomyocytes via increasing expressions of caspase 9 and caspase 3 (Figure 10). Our results implicate that DOX-induced apoptotic death of cardiac myocytes is the most direct cause of DOX-induced cardiotoxicity. As DOX treatment is prone to the generation of ROS, it may trigger the intrinsic mitochondrial-dependent apoptotic pathway. ${ }^{32}$ ROS produced in the mitochondria leads to release of Cyto-C and then activates the initiator caspase 9. Caspase 9 in turn activates the effector, and caspase 3 then initiates the apoptotic degradation. ${ }^{33}$ The combined effect of oxidative stress, inflammation, and apoptosis finally cause myofibrillar degeneration in the DOX-treated mice. To search for a safe and effective remedy to reverse DOX-induced cardiotoxicity remains a critical issue for cancer patients undergoing chemotherapy.

Nowadays, many natural extracts have been selected as a protective strategy against DOX-induced cardiotoxicity. For example, chrysin has been proven to protect against DOX-induced cardiotoxicity because of its multifunctional actions as an antioxidant, anti-inflammatory, anti-apoptotic, and anti-cancer agent. ${ }^{34-35}$ In the same way, we selected the herbal formula B307 as a protective strategy against DOXinduced cardiotoxicity because it has been widely used to enhance cardiovascular function and muscle strength. Our results had shown that survival rate and body weight of DOX-treated mice were obviously improved under oral B307 treatment (Figure 3). Furthermore, cardiac function and blood circulation in the DOX-treated mice were obviously improved under oral B307 treatment (Figures 4 and 5). Amelioration of blood circulation in the DOX-treated mice under oral B307 treatment may be attributed to enhancing expressions of eNOS in the heart tissue (Figure 5). As confirmed by histopathological examination, we observed that cardiac fibrosis deposition in the DOX-treated mice was alleviated under oral B307 treatment (Figure 5). In this study, we observed that cardiac oxidative damage in DOX-treated mice was alleviated under oral B307 treatment because cardiac expressions of SOD2 were significantly enhanced, but cardiac expressions of 3-NT were significantly suppressed (Figure 6). Also, the reduction of blood ROS production was observed in DOX-treated mice under oral B307 treatment (Figure 6). Moreover, we observed that cardiac inflammatory effects in DOX-treated mice under oral B307 treatment were alleviated by reducing expressions of NFKB1 (p50 and its precursor, p105) and hence inhibited the downstream inflammatory cascade as evidenced by decreasing the expression levels of TNF- $\alpha$. It is therefore clear that B307 treatment provides satisfactory anti-inflammatory effects (Figure 7). The anti-apoptotic effect of B307 might also be attributed to ROS scavenging capability. We observed that the ratio of Bcl-2 to Bax was significantly enhanced in DOX-treated mice under oral B307 treatment. Also, we observed that cardiac expressions of Cyto-C, Bax, calpain, 


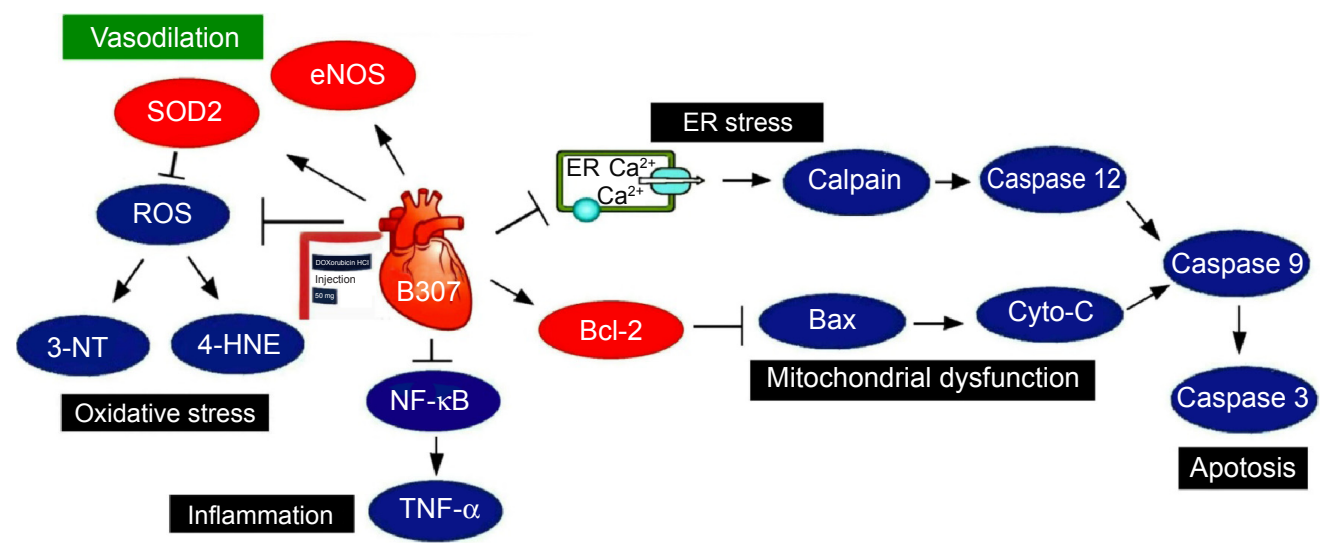

Figure I I The schematic diagram illustrates that oral treatment with the herbal formula B307 alleviates cardiotoxicity in doxorubicin-treated mice via suppressing hypoxia, oxidative stress, inflammation, and apoptosis in the heart tissue.

Abbreviations: eNOS, endothelial nitric oxide synthase; SOD2, superoxide dismutase 2; ROS, reactive oxygen species; 3-NT, neurotrophin-3; 4-HNE, 4-hydroxynonenal;

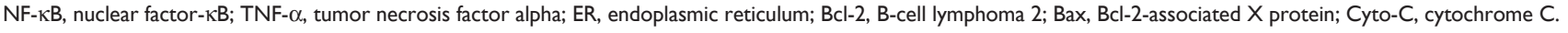

caspase 12 , caspase 9 , and caspase 3 were significantly suppressed in DOX-treated mice under oral B307 treatment. It is well known that caspases 9 and 3 also play key roles in cardiac apoptosis. ${ }^{36}$ Caspase 9 has been linked to the intrinsic mitochondrial death pathway. The release of Cyto-C from the mitochondria may trigger the activation of pro-caspase 9 (49 kDa), and cleaved caspase $9(39 \mathrm{kDa})$ can activate the downstream effector caspases, such as caspase 3, which eventually lead to apoptosis. Here, we observed that expressions of pro-caspase $9(49 \mathrm{kDa})$ and cleaved caspase 9 $(39 \mathrm{kDa})$ in the mice were significantly increased under DOX treatment but were significantly reduced under oral B307 treatment (Figure 10B). Furthermore, pro-caspase 3 $(35 \mathrm{kDa})$ and cleaved caspase $9(19 \mathrm{kDa})$ in the mice were significantly increased under DOX treatment but were significantly reduced under oral B307 treatment (Figure 10C). Based on the aforementioned discussion, we suggested that both mitochondrial dysfunction and ER stress-related apoptosis can be involved in cardiac pathogenesis of DOX treatment, while apoptosis damage in cardiac tissue of DOX-treated mice can be ameliorated under oral B307 treatment. Caspases 9 and 3 play the key role in cardiac apoptosis. ${ }^{36}$ Caspase 9 has been linked to the intrinsic mitochondrial death pathway. The release of Cyto- $\mathrm{C}$ from the mitochondria may trigger the activation of pro-caspase $9(49 \mathrm{kDa})$, and cleaved caspase $9(39 \mathrm{kDa})$ can activate the downstream effector caspases, such as caspase 3, which eventually lead to apoptosis. Here, we observed that expressions of pro-caspase $9(49 \mathrm{kDa})$ and cleaved caspase 9 $(39 \mathrm{kDa})$ of mice were significantly increased under DOX treatment, but were significantly reduced under oral B307 treatment (Figure 10B). Furthermore, pro-caspase 3 (35 kDa) and cleaved caspase $9(19 \mathrm{kDa})$ of mice were significantly increased under DOX treatment, but were significantly reduced under oral B307 treatment (Figure 10C). Based on the aforementioned discussion, we suggested that both mitochondrial dysfunction and ER stress-related apoptosis can be involved in cardiac pathogenesis of DOX treatment, while apoptosis damage in cardiac tissue of DOX-treated mice can be ameliorated under oral B307 treatment.

As shown in Figure 11, our studies demonstrated the potential for the herbal formula B307 in providing cardioprotective effects in DOX-induced acute cardiotoxicity. The main mechanisms underlying this cardioprotection could be primarily through suppressing cardiac oxidative stress, inflammation, and apoptosis in heart tissue. We have observed that there was no significant variation in Huh7 cancer cells between DOX only and DOX plus B307 treatment. Thus, we suggested that administration of the herbal formula B307 may provide a potential alternative treatment to fatal cardiac complication of DOX chemotherapy.

\section{Acknowledgments}

The authors thank Sun-Ten Pharmaceutical Company for the technical assistance. This work was supported by the IndustryUniversity Cooperative Grant of Brion Research Institute of Taiwan, Top University Project of National Taiwan Normal University (NTNU), and the Transnational Research Centers Grant (103T3040B04) from NTNU. The funding agency had no role in the study design, data collection/analysis, and the decision to publish the manuscript.

\section{Disclosure}

The authors declare no conflicts of interest in this work. 


\section{References}

1. Sant M, Allemani C, Santaquilani M, et al. EUROCARE working group. EUROCARE-4. Survival of cancer patients diagnosed in 1995-1999. Results and commentary. Eur J Cancer. 2009;45(6):931-991.

2. Smith MA, Seibel NL, Altekruse SF, et al. Outcomes for children and adolescents with cancer: challenges for the twenty-first century. J Clin Oncol. 2010;28(15):2625-2634.

3. Hayek ER, Speakman E, Rehmus E. Acute doxorubicin cardiac toxicity. N Engl J Med. 2005;352(23):2456-2457.

4. Minotti G, Menna P, Salvatorelli E, et al. Anthracyclines: molecular advances and pharmacologic developments in antitumor activity and cardiac toxicity. Pharmacol Rev. 2004;56(2):185-229.

5. Chen Y, Jungsuwadee P, Vore M, et al. Collateral damage in cancer chemotherapy: oxidative stress in non targeted tissues. Mol Interv. 2007; 7(3): 147-156.

6. Goormaghtigh E, Huart P, Praet M, et al. Structure of the adriamycincardiolipin complex role in mitochondrial toxicity. Biophys Chem. 1990;35(2-3):247-257.

7. Mantawy EM, El-Bakly WM, Esmat A, et al. Chrysin alleviates acute doxorubicin cardiac toxicity in rats via suppression of oxidative stress, inflammation and apoptosis. Eur J Pharmacol. 2014;728:107-118.

8. Barbaric M, Miskovic K, Bojic M, et al. Chemical composition of the ethanolic propolis extracts and its effect on HeLa cells. J Ethnopharmacol. 2011;135(3):772-778.

9. Chen XC, Zhu YG, Zhu LA, et al. Ginsenoside Rg1 attenuates dopamine-induced apoptosis in $\mathrm{PC} 12$ cells by suppressing oxidative stress. Eur J Pharmacol. 2003;473(1):1-7.

10. Gu B, Nakamichi N, Zhang WS, et al. Possible protection by notoginsenoside R1 against glutamate neurotoxicity mediated by N-methylD-aspartate receptors composed of an NR1/NR2B subunit assembly. J Neurosci Res. 2009;87(9):2145-2156.

11. Lee JS, Song JH, Sohn NW, et al. Inhibitory effects of ginsenoside Rb1 on neuroinflammation following systemic lipopolysaccharide treatment in mice. Phytother Res. 2012;27(9):1270-1276.

12. Liang $\mathrm{W}, \mathrm{Ge} \mathrm{S}$, Yang $\mathrm{L}$, et al. Ginsenosides Rb1 and Rg1 promote proliferation and expression of neurotrophic factors in primary Schwann cell cultures. Brain Res. 2010;1357:19-25.

13. Lin WM, Zhang YM, Moldzio R, et al. Ginsenoside Rb attenuates neuroinflammation of dopaminergic cells in culture. J Neural Transm Suppl. 2007;72:105-112.

14. Wang Y, Feng Y, Fu Q, et al. Panax notoginsenoside Rb1 ameliorates Alzheimer's disease by regulating brain-derived neurtotrophic factor and downregulating Tau protein expression. Exp Ther Med. 2013;6(3): 826-830.

15. Tam WY, Chook P, Qiao M, et al. The efficacy and tolerability of adjunctive alternative herbal medicine (Salvia miltiorrhiza and Pueraria lobata) on vascular function and structure in coronary patients. J Altern Complement Med. 2009;15(4):415-421.

16. Sieveking DP, Woo KS, Fung KP, et al. Chinese herbs Danshen and Gegen modulate key early atherogenic events in vitro. Int $J$ Cardiol. 2005;105(1):40-45.

17. Wu L, Qiao H, Li Y, et al. Protective roles of puerarin and Danshensu on acute ischemic myocardial injury in rats. Phytomedicine. 2007; 14(10):652-658.

18. Wu CH, Lai XJ, Cheng CJ, et al. Novel applications of digital holographic microscopy in therapeutic evaluation of Chinese herbal medicines. Appl Opt. 2014;53:G192-G197.

OncoTargets and Therapy

\section{Publish your work in this journal}

OncoTargets and Therapy is an international, peer-reviewed, open access journal focusing on the pathological basis of all cancers, potential targets for therapy and treatment protocols employed to improve the management of cancer patients. The journal also focuses on the impact of management programs and new therapeutic agents and protocols on

Submit your manuscript here: http://www.dovepress.com/oncotargets-and-therapy-journal
19. Wang SE, Lin CL, Hsu CH, et al. Treatment of a herbal formula B401 enhances neuroprotection and angiogenesis in the R6/2 mouse model of Huntington's disease. Drug Des Dev Ther. 2015;9:887-900.

20. Chien CT, Fan SC, Lin SC, et al. Glucagon-like peptide-1 receptor agonist activation ameliorates venous thrombosis-induced arteriovenous fistula failure in chronic kidney disease. Thromb Haemost. 2014; 112(5):1051-1064.

21. Fouad AA, Yacoubi MT. 2011. Mechanisms underlying the protective effect of eugenol in rats with acute doxorubicin cardiac toxicity. Arch Pharm Res. 2011;34(5):821-828.

22. Simunek T, Šterba M, Popelova O, et al. Anthracycline-induced cardiac toxicity: overview of studies examining the roles of oxidative stress and free cellular iron. Pharmacol Rep. 2009;61(1):154-171.

23. Doroshow JH, Locker GY, Myers CE. Enzymatic defenses of the mouse heart against reactive oxygen metabolites: alterations produced by doxorubicin. J Clin Invest. 1980;65(1):128-135.

24. Chaiswing L, Cole MP, St Clair DK, et al. Oxidative damage precedes nitrative damage in adriamycin-induced cardiac mitochondrial injury. Toxicol Pathol. 2004;32(5):536-547.

25. Hou G, Dick R, Abrams GD, et al. Tetrathiomolybdate protects against cardiac damage by doxorubicin in mice. J Lab Clin Med. 2005;146(5): 299-303.

26. Abd El-Aziz TA, Mohamed RH, Pasha HF, et al. Catechin protects against oxidative stress and inflammatory-mediated cardiotoxicity in adriamycin-treated rats. Clin Exp Med. 2012;12(4):233-240.

27. Kalay N, Basar E, Ozdogru I, et al. Protective effects of carvedilol against anthracycline-induced cardiomyopathy. J Am Coll Cardiol. 2006;48(11):2258-2262.

28. Reeve JL, Szegezdi E, Logue SE, et al. Distinct mechanisms of cardiomyocyte apoptosis induced by doxorubicin and hypoxia converge on mitochondria and are inhibited by Bcl-xL. J Cell Mol Med. 2007;11(3): 509-520.

29. Kunisada K, Tone E, Negoro S, et al. Bcl-xl reduces doxorubicin induced myocardial damage but fails to control cardiac gene downregulation. Cardiovasc Res. 2002;53(4):936-943.

30. Desagher S, Martinou JC. Mitochondria as the central control point of apoptosis. Trends Cell Biol. 2000;10(9):369-377.

31. Machado NG, Alves MG, Carvalho RA, et al. Mitochondrial involvement in cardiac apoptosis during ischemia and reperfusion: can we close the box? Cardiovasc Toxicol. 2009;9(4):211-227.

32. Green PS, Leeuwenburgh C. Mitochondrial dysfunction is an early indicator of doxorubicin-induced apoptosis. Biochim Biophys Acta. 2002; 1588(1):94-101.

33. Nicholson DW, Thornberry NA. Apoptosis. Life and death decisions. Science. 2003;299(5604):214-215.

34. Mantawy EM, El-Bakly WM, Esmat A, et al. Chrysin alleviates acute doxorubicin cardiac toxicity in rats via suppression of oxidative stress, inflammation and apoptosis. Eur J Pharmacol. 2014;728:107-118.

35. Sultana S, Verma K, Khan R. Nephroprotective efficacy of chrysin against cisplatin-induced toxicity via attenuation of oxidative stress. J Pharm Pharmacol. 2012;64(6):872-881.

36. Machado NG, Alves MG, Carvalho RA, et al. Mitochondrial involvement in cardiac apoptosis during ischemia and reperfusion: can we close the box? Cardiovasc Toxicol. 2009;9(4):211-227.

patient perspectives such as quality of life, adherence and satisfaction The manuscript management system is completely online and includes a very quick and fair peer-review system, which is all easy to use. Visit http://www.dovepress.com/testimonials.php to read real quotes from published authors. 